\title{
Insights into the emission of the blazar 1ES 1011+496 through unprecedented broadband observations during 2011 and 2012
}

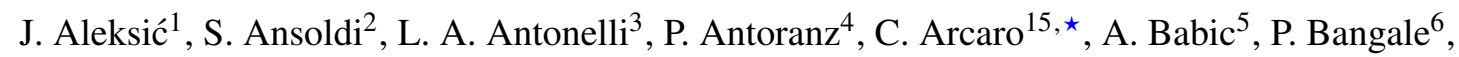
U. Barres de Almeida ${ }^{6,31, \star}$, J. A. Barrio ${ }^{7}$, J. Becerra González ${ }^{8,25}$, W. Bednarek ${ }^{9}$, E. Bernardini ${ }^{10}$, B. Biasuzzi ${ }^{2}$, A. Biland ${ }^{11}$, O. Blanch ${ }^{1}$, S. Bonnefoy ${ }^{7}$, G. Bonnoli ${ }^{3}$, F. Borracci ${ }^{6}$, T. Bretz ${ }^{12,26}$, E. Carmona ${ }^{13}$, A. Carosi ${ }^{3}$, P. Colin ${ }^{6}$, E. Colombo ${ }^{8}$, J. L. Contreras ${ }^{7}$, J. Cortina ${ }^{1}$, S. Covino ${ }^{3}$, P. Da Vela ${ }^{4}$, F. Dazzi ${ }^{6}$, A. De Angelis ${ }^{2}$, G. De Caneva ${ }^{10}$, B. De Lotto ${ }^{2}$, E. de Oña Wilhelmi ${ }^{14}$, C. Delgado Mendez ${ }^{13}$, F. Di Pierro ${ }^{3}$, D. Dominis Prester ${ }^{5}$, D. Dorner ${ }^{12}$, M. Doro ${ }^{15}$, S. Einecke ${ }^{16}$, D. Eisenacher ${ }^{12}$, D. Elsaesser ${ }^{12}$, A. Fernández-Barral ${ }^{1}$, D. Fidalgo ${ }^{7}$, M. V. Fonseca ${ }^{7}$, L. Font ${ }^{17}$, K. Frantzen ${ }^{16}$, C. Fruck $^{6}$, D. Galindo ${ }^{18}$, R. J. García López ${ }^{8}$, M. Garczarczyk ${ }^{10}$, D. Garrido Terrats ${ }^{17}$, M. Gaug ${ }^{17}$, N. Godinović ${ }^{5}$, A. González Muñoz ${ }^{1}$, S. R. Gozzini ${ }^{10}$, D. Hadasch ${ }^{14,27}$, Y. Hanabata ${ }^{19}$, M. Hayashida ${ }^{19}$, J. Herrera ${ }^{8}$, J. Hose $^{6}$, D. Hrupec ${ }^{5}$, W. Idec ${ }^{9}$, V. Kadenius, H. Kellermann ${ }^{6}$, M. L. Knoetig ${ }^{11}$, K. Kodani ${ }^{19}$, Y. Konno ${ }^{19}$, J. Krause ${ }^{6}$, H. Kubo ${ }^{19}$, J. Kushida ${ }^{19}$, A. La Barbera ${ }^{3}$, D. Lelas ${ }^{5}$, N. Lewandowska ${ }^{12}$, E. Lindfors ${ }^{20,28}$, S. Lombardi ${ }^{3}$, F. Longo ${ }^{2}$, M. López ${ }^{7}$, R. López-Coto ${ }^{1}$, A. López-Oramas ${ }^{1}$, E. Lorenz ${ }^{6}$, I. Lozano ${ }^{7}$, M. Makariev ${ }^{21}$, K. Mallot ${ }^{10}$, G. Maneva ${ }^{21}$, K. Mannheim ${ }^{12}$, L. Maraschi ${ }^{3}$, B. Marcote $^{18}$, M. Mariotti ${ }^{15}$, M. Martínez ${ }^{1}$, D. Mazin ${ }^{6}$, U. Menzel ${ }^{6}$, J. M. Miranda ${ }^{4}$, R. Mirzoyan ${ }^{6}$, A. Moralejo ${ }^{1}$, P. Munar-Adrover ${ }^{18}$, D. Nakajima ${ }^{19}$, V. Neustroev ${ }^{20}$, A. Niedzwiecki ${ }^{9}$, M. Nievas Rosillo ${ }^{7}$, K. Nilsson ${ }^{20,28}$, K. Nishijima ${ }^{19}$, K. Noda ${ }^{6}$, R. Orito ${ }^{19}$, A. Overkemping ${ }^{16}$, S. Paiano ${ }^{15, \star}$, M. Palatiello ${ }^{2}$, D. Paneque ${ }^{6}$, R. Paoletti ${ }^{4}$, J. M. Paredes ${ }^{18}$, X. Paredes-Fortuny ${ }^{18}$, M. Persic ${ }^{2,29}$, J. Poutanen ${ }^{20}$, P. G. Prada Moroni ${ }^{22}$, E. Prandini ${ }^{11,30}$, I. Puljak ${ }^{5}$, R. Reinthal ${ }^{20}$, W. Rhode ${ }^{16}$, M. Ribó ${ }^{18}$, J. Rico ${ }^{1}$, J. Rodriguez Garcia ${ }^{6}$, T. Saito ${ }^{19}$, K. Saito ${ }^{19}$, K. Satalecka ${ }^{7}$, V. Scalzotto ${ }^{15}$, V. Scapin ${ }^{7}$, T. Schweizer ${ }^{6}$, S. N. Shore ${ }^{22}$, A. Sillanpää ${ }^{20}$, J. Sitarek ${ }^{1}$, I. Snidaric ${ }^{5}$, D. Sobczynska ${ }^{9}$, A. Stamerra ${ }^{3}$, T. Steinbring ${ }^{12}$, M. Strzys ${ }^{6}$, L. Takalo ${ }^{20}$, H. Takami ${ }^{19}$,

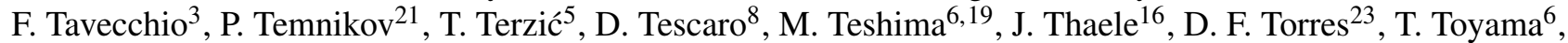
A. Treves ${ }^{24}$, P. Vogler ${ }^{11}$, M. Will ${ }^{8}$, R. Zanin ${ }^{18}$, S. Buson ${ }^{15}$, F. D'Ammando ${ }^{32}$, A. Lähteenmäki ${ }^{33,34}$, T. Hovatta ${ }^{35,33}$, Y. Y. Kovalev ${ }^{36,37}$, M. L. Lister ${ }^{38}$, W. Max-Moerbeck ${ }^{39}$, C. Mundell ${ }^{40}$, A. B. Pushkarev ${ }^{41,42,37}$, E. Rastorgueva-Foi ${ }^{33}$, A. C. S. Readhead ${ }^{35}$, J. L. Richards ${ }^{37}$, J. Tammi ${ }^{33}$, D. A. Sanchez ${ }^{43}$, M. Tornikoski ${ }^{33}$, T. Savolainen ${ }^{37}$, and I. Steele ${ }^{40}$

\section{(Affiliations can be found after the references)}

Received 12 August 2015 / Accepted 10 February 2016

\section{ABSTRACT}

Context. 1ES 1011+496 $(z=0.212)$ was discovered in very high-energy (VHE, $E>100 \mathrm{GeV}) \gamma$ rays with MAGIC in 2007. The absence of simultaneous data at lower energies led to an incomplete characterization of the broadband spectral energy distribution (SED).

Aims. We study the source properties and the emission mechanisms, probing whether a simple one-zone synchrotron self-Compton (SSC) scenario is able to explain the observed broadband spectrum.

Methods. We analyzed data in the range from VHE to radio data from 2011 and 2012 collected by MAGIC, Fermi-LAT, Swift, KVA, OVRO, and Metsähovi in addition to optical polarimetry data and radio maps from the Liverpool Telescope and MOJAVE.

Results. The VHE spectrum was fit with a simple power law with a photon index of $3.69 \pm 0.22$ and a flux above $150 \mathrm{GeV}$ of $(1.46 \pm 0.16) \times$ $10^{-11} \mathrm{ph} \mathrm{cm}^{-2} \mathrm{~s}^{-1}$. The source 1ES $1011+496$ was found to be in a generally quiescent state at all observed wavelengths, showing only moderate variability from radio to X-rays. A low degree of polarization of less than $10 \%$ was measured in optical, while some bright features polarized up to $60 \%$ were observed in the radio jet. A similar trend in the rotation of the electric vector position angle was found in optical and radio. The radio maps indicated a superluminal motion of $1.8 \pm 0.4 c$, which is the highest speed statistically significant measured so far in a high-frequency-peaked BL Lac.

Conclusions. For the first time, the high-energy bump in the broadband SED of 1ES 1011+496 could be fully characterized from $0.1 \mathrm{GeV}$ to $1 \mathrm{TeV}$, which permitted a more reliable interpretation within the one-zone SSC scenario. The polarimetry data suggest that at least part of the optical emission has its origin in some of the bright radio features, while the low polarization in optical might be due to the contribution of parts of the radio jet with different orientations of the magnetic field with respect to the optical emission.

Key words. BL Lacertae objects: individual: 1ES 1011+496 - galaxies: active - galaxies: jets - radiation mechanisms: non-thermal gamma rays: galaxies

\footnotetext{
^ Coresponding authors: C. Arcaro, e-mail: cornelia.arcaro@pd.infn.it; U. Barres de Almeida, e-mail: ulisses@cbpf.br S. Paiano, e-mail: simona.paiano@pd.infn.it
} 


\section{Introduction}

Blazars are a subclass of radio-loud active galactic nuclei (AGNs) with their relativistic particle jets closely aligned to the line of sight of the observer. They are highly variable at nearly all wavelengths at various timescales, and their emission is dominated by a non-thermal continuum spanning from radio to VHE $\gamma$ rays which is assumed to be produced within the jets and boosted by beaming (e.g., Urry \& Padovani 1995; Ghisellini 2000). The spectral energy distribution (SED) of blazars shows two distinct broad components: a low-energy bump in the optical to X-ray range that is commonly associated with synchrotron emission of electrons and a high-energy bump in the $\gamma$-ray band. The origin of the latter is usually explained by leptonic models in terms of inverse Compton scattering of synchrotron photons (e.g., Tavecchio et al. 1998; Katarzyński et al. 2001) or external photons (e.g., Sikora et al. 1994), but hadronic emission models have also been proposed (e.g., Mannheim 1993).

Based on their optical spectra (e.g., Stickel 1991), blazars are divided into two classes: flat spectrum radio quasars (FSRQs) that show broad emission lines and BL Lac objects characterized by the weakness or even absence of such lines. The latter were further subdivided into low- and high-energy cutoff BL Lacs (LBLs, HBLs) depending on the radio-to-X-ray spectral slope, which gives the SED's synchrotron peak position (Padovani \& Giommi 1995; Urry \& Padovani 1995). An alternative definition was given in Abdo et al. (2010a), where blazars are classified as low, intermediate, and high synchrotron-peaked blazars (LSP, ISP, HSP) based on the location of the synchrotron peak. Later on, Spurio (2014) defined LBLs, IBLs, and HBLs according to the synchrotron peak positions given in Abdo et al. (2010a) for LSPs, ISPs, and HSPs. Since blazars show flux variability at all wavelengths at different timescales ranging down to minutes, simultaneous observations are a useful tool for studying the overall SED and constraining the physical processes that govern the emission in their jets.

1ES 1011+496 $(\mathrm{RA}=10: 15: 04.14$, Dec $=49: 26: 00.70$; $\mathrm{J} 2000$ ) is a blazar located at redshift $z=0.212 \pm 0.002$ (Albert et al. 2007a) ${ }^{1}$ classified as an HBL based on the radio-to-Xray ratio (Padovani \& Giommi 1995; Donato et al. 2001) and the presence of a featureless optical spectrum (Wisniewski et al. 1986). It was suggested as a VHE $\gamma$-ray candidate with a predicted integral flux of $0.12 \times 10^{-11} \mathrm{ph} \mathrm{cm}^{-2} \mathrm{~s}^{-1}$ above $300 \mathrm{GeV}$ by Costamante \& Ghisellini (2002). From 1996 to 2006 the source was the target of several VHE $\gamma$-ray observations by HEGRA (Aharonian et al. 2004), the Whipple Observatory $10 \mathrm{~m} \gamma$-ray telescope (Fegan et al. 2005), and MAGIC (Albert et al. 2008a; Aleksić et al. 2011) yielding only integral flux upper limits. In 2007 MAGIC detected the source first in the VHE regime (Albert et al. 2007a) and subsequently detected it in 2008 (Ahnen et al. 2015). Considering the first two years of Fermi-LAT observations reported in the second Fermi-LAT catalog (2FGL; Nolan et al. 2012), 1ES 1011+496 is associated with the source 2FGL J1015.1+4925, which has been observed with an integral flux of $(4.4 \pm 0.3) \times 10^{-8} \mathrm{ph} \mathrm{cm}^{-2} \mathrm{~s}^{-1}$ $(100 \mathrm{MeV}-100 \mathrm{GeV})$. The high-energy (HE, $100 \mathrm{MeV}<E<$ $100 \mathrm{GeV}) \gamma$-ray spectrum was able to be fit with a log parabola of the form $\frac{\mathrm{d} N}{\mathrm{~d} E}=N_{0}\left(\frac{E}{E_{\mathrm{b}}}\right)^{-\left(\alpha+\beta \log \left(E / E_{\mathrm{b}}\right)\right)}$, where $N_{0}=(1.01 \pm$ $0.04) \times 10^{-11} \mathrm{ph} \mathrm{cm}^{-2} \mathrm{~s}^{-1} \mathrm{MeV}^{-1}$ and $\alpha=1.72 \pm 0.04$ denote the normalized flux and the spectral index, respectively, at the

\footnotetext{
1 This redshift corresponds to a luminosity distance of $1.04 \mathrm{Gpc}$ for contemporary cosmology parameters, i.e., $H_{0}=71 \mathrm{~km} \mathrm{~s}^{-1} \mathrm{Mpc}^{-1}, \Omega_{\Lambda}=$ $0.73, \Omega_{\mathrm{c}}=0.27$ (Spergel et al. 2003).
}

pivot energy $E_{\mathrm{b}}=812.6 \mathrm{MeV}$, and $\beta=0.075 \pm 0.019$ is a measure of the spectral curvature. In the third Fermi-LAT source catalog (3FGL; Acero et al. 2015) an integral flux of (5.1 \pm $0.2) \times 10^{-8} \mathrm{ph} \mathrm{cm}^{-2} \mathrm{~s}^{-1}(100 \mathrm{MeV}-100 \mathrm{GeV})$ was reported. A simple power-law fit with a photon index of $1.83 \pm 0.02$ was sufficient to describe the spectrum obtained from four years of Fermi operation. Above $10 \mathrm{GeV}$ the spectrum is described well by a simple power-law fit with a photon index of $2.28 \pm 0.16$ and the integral flux corresponds to $(7.87 \pm 0.89) \times 10^{-8} \mathrm{ph} \mathrm{cm}^{-2} \mathrm{~s}^{-1}$ $(10-500 \mathrm{GeV})$ as reported in the first Fermi High-energy LAT catalog (1FHL, $E>10 \mathrm{GeV}$; Ackermann et al. 2013).

Based on archival multiwavelength (MWL) data, Ahnen et al. (2015) discuss that the source's characteristics resemble those of an IBL during low and medium flux states, whereas at high states they are similar to an HBL, concluding therefore that the source seems to be a borderline case between IBL and HBL.

In blazar studies the polarization represents a powerful tool for distinguishing between the competing physical models regarding the particle and seed photon populations responsible for their VHE $\gamma$-ray emission (e.g., Pavlidou et al. 2013). Furthermore, the study of the position angle provides information on the orientation of the magnetic field of the emission region thus helping to understand the state of the plasma and the particle population in the location of emission (e.g., Barres de Almeida et al. 2010). In some cases, large changes in polarization angle have been associated with $\gamma$-ray flares (e.g., Abdo et al. 2010b), but the link between rotations in polarization angle and high-energy activity is still under study (e.g., Blinov et al. 2015).

In this paper we report for the first time MAGIC stereo observations of 1ES 1011+496 carried out from 2011 to 2012, and provide a more accurate VHE $\gamma$-ray spectrum (Sect. 3.1) than those measured in 2007 (Albert et al. 2007a) and 2008 (Ahnen et al. 2015) when MAGIC operated with a single telescope. We discuss the MWL variability (Sect. 3.2) of the source based on simultaneous data in $\mathrm{HE} \gamma$ rays from the Fermi Large Area Telescope (LAT), in X-rays and UV bands by Swift (XRT/UVOT), in the optical $R$-band by the KVA telescope, and in the radio band respectively at 37 and $15 \mathrm{GHz}$ by the Metsähovi and OVRO telescopes. The individual instruments involved in these MWL observations are described in Sect. 2 including information on the observations and the data analysis. We combine these MWL observations with optical polarimetry data from the Liverpool Telescope and multi-epoch radio maps from $\mathrm{MOJAVE}^{2}$ in order to put further constraints on the site and structure of the VHE $\gamma$-ray emission region. We model the broadband SED compiled from these MWL observations assuming a one-zone synchrotron self-Compton (SSC) scenario (Sect. 4). Our conclusions are summarized in Sect. 5.

\section{Observations and data analysis}

\subsection{MAGIC}

Since 2009 MAGIC has been operating as a stereoscopic system of two $17 \mathrm{~m}$ Imaging Atmospheric Cherenkov Telescopes, MAGIC I and MAGIC II, that are located at the Roque de Los Muchachos, La Palma, Canary Islands $\left(28.8^{\circ} \mathrm{N}, 17.9^{\circ} \mathrm{W}\right.$, $2225 \mathrm{~m}$ a.s.1.). Owing to its low energy threshold (as low as

\footnotetext{
2 Monitoring of Jets in Active Galactic Nuclei with VLBA Experiments (Lister et al. 2009).
} 
$60 \mathrm{GeV}$ in normal trigger mode) and high sensitivity ${ }^{3}$, MAGIC is well suited for VHE $\gamma$-ray observations of blazars. In 2011 July and in 2012 July, a further upgrade of MAGIC was carried out by decommissioning the old MAGIC I camera and MUX readout electronics with the aim to further improve the performance of the MAGIC stereo system that was limited by the smaller trigger region and the slightly lower light conversion efficiency of the MAGIC I camera (Mazin et al. 2013).

In a first phase in late 2011, the readout system of the MAGIC I telescope was upgraded to a digitizing system based on the domino-ring-sampler $\left(\mathrm{DRS}^{4}\right)$ version 4. Compared to the DRS-2 chip, previously used in MAGIC II, the dead time has been significantly reduced to less than $1 \%$ (Sitarek et al. 2013). In the course of this hardware change, the MAGIC II readout system was also updated to this latest chip version. The dominant sources of systematic uncertainties are not related to the readout system, but rather to the spectral reflectivity of the mirrors, the camera photon detection efficiency, and the atmospheric characterization; and hence the prescription reported in Aleksić et al. (2012) is still valid for the 2012 data

1ES 1011+496 was observed with MAGIC during dark nights and under moderate Moon conditions at zenith angles spanning from $24^{\circ}$ to $50^{\circ}$. In 2011 , observations were performed during 12 individual nights between the end of February and beginning of April for a total of $\sim 13 \mathrm{~h}$, while observations in 2012 were performed from the end of January until the middle of May over 33 nights for a total of $\sim 23 \mathrm{~h}$ with the upgraded readout system.

The total effective observation time after corrections for the dead time of the readout system is $\sim 30.6 \mathrm{~h}$. Observations were performed in the so-called wobble mode (Fomin et al. 1994) which means that the two telescopes alternated every 20 min between two (in 2011) or four (in 2012) sky positions with an offset of 0.4 from the source. The data were analyzed using the MAGIC analysis and reconstruction software (MARS) package (Zanin et al. 2013) that was adapted to stereoscopic observations. The image cleaning was performed according to Aliu et al. (2009).

The images were parametrized in each telescope individually according to the prescription of Hillas (1985). For the reconstruction of the shower arrival direction the random forest regression method (RF DISP method; Aleksić et al. 2010) with the implementation of stereoscopic parameters such as the impact distance of the shower on the ground was used (Lombardi et al. 2011). The $\gamma /$ hadron separation was performed by using the random forest method (Albert et al. 2008c) which is based on both individual image parameters from each telescope and stereoscopic information such as the shower impact point and the shower height maximum. Energy look-up tables were used for the energy reconstruction. Further details on the stereo MAGIC analysis can be found in Aleksić et al. (2012).

For sources with VHE $\gamma$-ray spectra similar to that of the Crab Nebula, the sensitivity of the MAGIC stereo system is best above 250-300 GeV. For sources with spectral shapes softer than that of the Crab Nebula, the best performance occurs at slightly lower energies. Consequently, we chose $150 \mathrm{GeV}$ as the minimum energy to report signal significances and $\gamma$-ray fluxes in light curves, while for the spectral analysis, in order to use all

\footnotetext{
Better than $0.8 \%$ of the Crab Nebula flux in $50 \mathrm{~h}$ of observing time above $290 \mathrm{GeV}$ (Aleksić et al. 2012) in stereoscopic mode, while in mono mode the best sensitivity achieved above $250 \mathrm{GeV}$ was $2.2 \%$ of the Crab Nebula flux in 50 h (Albert et al. 2008b).

4 http://www.psi.ch/drs/
}

the available information, we also considered energies well below $150 \mathrm{GeV}$ where the analysis of the MAGIC data can still be performed (Aleksić et al. 2016).

\subsection{Fermi-LAT}

1ES 1011+496 has been observed by the pair-conversion telescope Fermi-LAT optimized for energies from $20 \mathrm{MeV}$ up to energies beyond $300 \mathrm{GeV}$ (Atwood et al. 2009). In survey mode the Fermi-LAT scans the entire sky every three hours. The data sample, which consists of observations from 2011 February 24 to April 7, and from 2012 January 1 to May 30, was analyzed with the standard analysis tool gtlike, part of the Fermi Science Tools software package (version 09-27-01) available from the Fermi Science Support Center ${ }^{5}$ We selected events of the CLEAN ${ }^{6}$ class with energies from $100 \mathrm{MeV}$ to $300 \mathrm{GeV}$ located in a circular region of interest (ROI) of $10^{\circ}$ radius centered on the position of 1ES 1011+496. Time intervals when the LAT boresight was rocked with respect to the local zenith by more than $52^{\circ}$ and events with a reconstructed angle with respect to the local zenith $>100^{\circ}$ were excluded. This latter selection was necessary to limit the contamination from $\gamma$ rays produced by interactions of cosmic rays with the upper atmosphere of the Earth. In addition, to correct the calculation of the exposure for the zenith cut, time intervals when any part of the ROI was observed at zenith angles $>100^{\circ}$ were excluded. For the $\gamma$-ray signal extraction, the background model included two components: a Galactic diffuse emission and an isotropic diffuse, provided by the publicly available files gal_2yearp7v6_trim_v0.fits and iso_p7v6clean.txt ${ }^{7}$. The model of the ROI also included sources from the 2FGL (Nolan et al. 2012), which are located within $15^{\circ}$ of $1 \mathrm{ES} 1011+496$. These sources, as well as the source of interest, were modeled with a power-law spectral shape. We first fitted the whole dataset considered in this paper and then used the resulting best-fit ROI model to produce the light curve and SED. In the light curve and SED fitting, the spectral parameters of sources within $10^{\circ}$ from our target were allowed to vary while those within $10^{\circ}-15^{\circ}$ were fixed to their initial values. During the spectral fitting, the normalizations of the background models were allowed to vary freely. Spectral parameters were estimated from $300 \mathrm{MeV}$ to $300 \mathrm{GeV}$ using an unbinned maximum likelihood technique (Mattox et al. 1996) taking into account the post-launch instrument response functions (specifically P7CLEAN_V6, Ackermann et al. 2012). When producing the SED and the light curves only the parameter of the source of interest were free to vary. The parameters of other sources in the ROI were kept fixed to average values found over the studied period.

During the MAGIC observing period, the source was not significantly detected on a daily basis. To ensure a good compromise between having a significant detection in most of the intervals and details on the temporal behavior of the source, the light curves were produced with weekly binning for the 2011 period, and with a three-day binning for the 2012 period (second panel from the top in Fig. 3). To produce the Fermi-LAT SED, simultaneous to the MAGIC observation periods, the previously

\footnotetext{
5 http://fermi.gsfc.nasa.gov/ssc/

6 The CLEAN class was chosen in this analysis since it ensures a higher signal-to-noise ratio with respect to the SOURCE class. For more information refer to http://fermi.gsfc.nasa.gov/ssc/ data/analysis/LAT_caveats_pass7.html.

7 http://fermi.gsfc.nasa.gov/ssc/data/access/lat/ BackgroundModels.html
} 
mentioned 2011 and 2012 time periods were combined to build an average SED using the fmerge ${ }^{8}$ HEASARC tool. Flux upper limits at the $95 \%$ confidence level were calculated for each time bin where the Test Statistic $\left(\mathrm{TS}^{9}\right)$ value for the source was below 9 . The systematic uncertainty on the flux is dominated by the systematic uncertainty on the effective area, which is estimated to be $10 \%$ at $100 \mathrm{MeV}$, decreasing to $5 \%$ at $560 \mathrm{MeV}$, and increasing to $10 \%$ at $10 \mathrm{GeV}$ (Ackermann et al. 2012). The systematic uncertainties are smaller than the statistical uncertainties of the data points in the light curves and spectra.

\subsection{Swift/XRT and Swift/UVOT}

The Swift satellite (Gehrels et al. 2004) performed four observations of 1ES 1011+496 between 2012 March 20 and 31 as part of a target of opportunity request for a dedicated MWL campaign. The observations were performed with all three onboard instruments: the X-ray Telescope (XRT; Burrows et al. 2005, 0.2-10.0 keV), the Ultraviolet Optical Telescope (UVOT; Roming et al. 2005, 170-600 nm), and the Burst Alert Telescope (BAT; Barthelmy et al. 2005, 15-150 keV). The hard X-ray flux of this source is below the sensitivity of the BAT instrument for the short exposures of these observations and so the data from this instrument are not used.

The XRT data were processed with standard procedures (xrtpipeline vQ.12.6), filtering, and screening criteria by using the Heasoft package (v6.13). The data were collected in photon counting mode, and only XRT event grades 0-12 were selected (according to the Swift nomenclature; Burrows et al. 2005). The XRT observations showed a source count rate $>0.5$ counts $\mathrm{s}^{-1}$ requiring a pile-up correction. Source events were extracted from an annular region with an inner radius of 5 pixels (estimated by means of the PSF fitting technique) and an outer radius of 30 pixels (1 pixel $\sim 2$ '!36). Background events were extracted within an annular region centered on the source with radii of 70 and 120 pixels. Ancillary response files were generated with xrtmkarf, and account for different extraction regions, vignetting, and PSF corrections. We used the spectral redistribution matrix v014 in the Calibration database ${ }^{10}$ (CALDB 20131220) maintained by HEASARC. The Swift/XRT spectra were rebinned in order to have at least 20 counts per energy bin. Considering the low number of photons collected (<200 counts) the spectrum collected on 2012 March 23 was rebinned with a minimum of 1 count per bin and the Cash statistic (Cash 1979) was used. A fit was performed with Xspec (v12.7.1) adopting an absorbed power-law model with free photon index using the photoelectric absorption model tbabs with a neutral hydrogen column fixed to its Galactic value $N_{\mathrm{H}}=8.38 \times$ $10^{19} \mathrm{~cm}^{-2}$ (Kalberla et al. 2005). During the Swift pointing the UVOT instrument observed 1ES $1011+496$ in the $V, B, U, W 1$, $M 2$, and $W 2$ photometric bands (Poole et al. 2008). The analysis was performed using the uvotsource tool to extract counts from a standard $00^{\prime \prime 5}$ radius source aperture. To calculate the

\footnotetext{
8 https://heasarc.gsfc.nasa.gov/ftools/caldb/help/ fmerge.txt

9 The Test Statistic value quantifies the probability of having a pointlike $\gamma$-ray source at the location specified. It corresponds roughly to the standard deviation squared assuming one degree of freedom (Mattox et al. 1996). The TS is defined as $-2 \log \left(L_{0} / L\right)$, where $L_{0}$ is the maximum likelihood value for a model without an additional source (i.e., the null hypothesis) and $L$ is the maximum likelihood value for a model with the additional source at the specified location.

${ }^{10}$ http://heasarc.gsfc.nasa.gov/docs/heasarc/caldb/ swift/
}

source flux, a correction for coincidence losses and a background subtraction was applied. The background counts were derived from a circular region of $10^{\prime \prime}$ radius in the source neighborhood. Conversion of magnitudes into dereddened flux densities was obtained by adopting the extinction value $E(B-V)=0.010$ from Schlafly \& Finkbeiner (2011), the mean Galactic extinction curve from Fitzpatrick (1999), and the magnitude-flux calibrations by Bessell et al. (1998).

\subsection{KVA and Liverpool telescopes}

The optical data were collected with the KVA telescopes ${ }^{11}$ located at the Roque de los Muchachos observatory on La Palma. They are operated under the Tuorla Blazar Monitoring Program $^{12}$, which runs as a support program to the MAGIC observations. The program started at the end of 2002 and uses the KVA telescope together with the Tuorla $1 \mathrm{~m}$ instrument (located in Finland) to monitor VHE $\gamma$-ray candidates (Costamante \& Ghisellini 2002) and known TeV blazars in the optical waveband. It is also used to alert MAGIC on high states of these objects in order to trigger follow-up VHE $\gamma$-ray observations. 1ES $1011+496$ was one of the objects on the original target list and has therefore been monitored regularly since the beginning of the program. The data presented here comprise 2011 and 2012 observations. Both KVA telescopes are operated remotely from Finland. The smaller of the two telescopes, a $35 \mathrm{~cm}$ Celestron, is used for photometric measurements, while the larger one $(60 \mathrm{~cm})$ is used for polarimetric observations of some of the brighter objects. The photometric measurements are performed in the optical $R$-band using differential photometry, i.e., the target and the calibrated comparison stars are recorded on the same CCD images (Fiorucci et al. 1998). The magnitudes of the source and comparison stars are measured via aperture photometry and are converted to fluxes applying the formula $F(\mathrm{Jy})=F_{0} \times 10^{-0.4 \mathrm{~m}}$, where $F_{0}$ is a filter-dependent zero point $\left(F_{0}=3080 \mathrm{Jy}\right.$ in the $R$-band, from Bessell 1979). In order to obtain the AGN core emission, contributions from the host galaxy and possible nearby stars that add to the overall measured flux have to be subtracted. In the case of $1 \mathrm{ES} 1011+496$, the host galaxy contribution is $(0.49 \pm 0.02) \mathrm{mJy}$ (Nilsson et al. 2007).

In 2012 the optical polarimetry data were taken from mid-March to the end of May with the fast readout imaging polarimeter RINGO 2 (Steele et al. 2010) mounted on the Liverpool telescope. The instrument is equipped with a hybrid $V+R$ filter consisting of a $3 \mathrm{~mm}$ Schott GG475 filter cemented to a $2 \mathrm{~mm}$ KG3 filter. The polarimeter uses a rotating polaroid with a frequency of $\sim 1 \mathrm{~Hz}$ that takes eight exposures of the source during a cycle. To determine the degree and angle of polarization, these exposures were synchronized with the phase of the polaroid (Mundell et al. 2013). The data was analyzed as in Aleksić et al. (2014a) using the standard procedures.

\subsection{Metsähovi and OVRO telescopes and the VLBA}

The $37 \mathrm{GHz}$ observations were performed with the $13.7 \mathrm{~m}$ diameter Metsähovi Radio Telescope ${ }^{13}$, a radome-enclosed paraboloid antenna situated in Finland, during the second half of the 2012 MWL campaign from mid-March to mid-May. Measurements were performed with a $1 \mathrm{GHz}$-band dual-beam receiver centered at $36.8 \mathrm{GHz}$, whose high electron mobility

\footnotetext{
11 http://www . astro.utu.fi/telescopes/60lapalma.htm

12 Project web page: http://users.utu.fi/kani/

13 http://metsahovi.aalto.fi/en/
} 
Table 1. VHE $\gamma$-ray spectrum of 1ES 1011+496 observed with MAGIC in 2007 (Albert et al. 2007a), 2008 (Ahnen et al. 2015), and between 2011 and 2012.

\begin{tabular}{cccccc}
\hline \hline Year & $\begin{array}{c}\text { Range } \\
{[\mathrm{GeV}]}\end{array}$ & $\begin{array}{c}f_{\text {0obs }} \\
{\left[10^{-10} \mathrm{ph} \mathrm{cm}^{-2} \mathrm{~s}^{-1} \mathrm{TeV}^{-1}\right]}\end{array}$ & $\Gamma_{\text {obs }}$ & $\Gamma_{\text {deabs }}$ & $\begin{array}{c}F(>200 \mathrm{GeV}) \\
{\left[10^{-11} \mathrm{ph} \mathrm{cm}^{-2} \mathrm{~s}^{-1}\right]}\end{array}$ \\
\hline 2007 & $150-590$ & $2.0 \pm 0.1$ & $4.0 \pm 0.5$ & $3.9 \pm 0.7$ & $1.58 \pm 0.32$ \\
2008 & $120-910$ & $1.8 \pm 0.5$ & $3.3 \pm 0.4$ & $2.2 \pm 0.4$ & $1.3 \pm 0.3$ \\
$2011 / 2012$ & $95-870$ & $1.33 \pm 0.06$ & $3.66 \pm 0.22$ & $3.0 \pm 0.3$ & $0.75 \pm 0.12$ \\
\hline
\end{tabular}

Notes. From left to right: year of observation, fit range, flux normalization $f_{0}$ at $200 \mathrm{GeV}$, spectral slopes $\Gamma_{\text {obs }}$ and $\Gamma_{\text {deabs }}$ from a simple power-law fit of the observed and deabsorbed spectrum using the EBL models from Kneiske et al. (2002) for 2007 and from Domínguez et al. (2011) for 2008 and 2011/2012 observations, respectively.

pseudomorphic transistor front end operates at room temperature. So-called ON-ON observations were performed where the source and the sky are alternated in each feed horn. The flux density scale was set by observations of DR 21 (a huge molecular cloud located in the constellation of Cygnus used as a standard candle for radio astronomy), whereas the sources NGC 7027, 3C 274, and 3C 84 were used as secondary calibrators. A detailed description of the data reduction and analysis can be found in Teraesranta et al. (1998). The error estimated in the flux density includes the contribution from the measurement rms and the uncertainty of the absolute calibration. Upper limits at $95 \%$ confidence level were calculated for each measurement with a signal-to-noise ratio of $S / N<4$.

Regular $15 \mathrm{GHz}$ observations of 1ES $1011+496$ were carried out using the OVRO (Owens Valley Radio Observatory) $40 \mathrm{~m}$ telescope (Richards et al. 2011), which is located in California. The center frequency of the receiver is $15 \mathrm{GHz}$ with a bandwidth of $3 \mathrm{GHz}$. The two sky beams are Dicke switched, and the source is alternated between the two beams in an ON-ON fashion to remove atmospheric and ground contamination. A noise level of approximately 3-4 mJy in quadrature with about $2 \%$ additional uncertainty, mostly due to pointing errors, is achieved in a $70 \mathrm{~s}$ integration period. Calibration is achieved using a temperature-stable diode noise source to remove receiver gain drifts. Occasional gaps in the data sampling are due to poor weather conditions or maintenance. The data were calibrated against 3C 286 with an assumed flux density of $3.44 \mathrm{Jy}$ at $15 \mathrm{GHz}$ (Baars et al. 1977) and analyzed via the pipeline described in Richards et al. (2011). The observations of 1ES $1011+496$ were carried out in the framework of a blazar monitoring program (Richards et al. 2011) measuring the source flux density twice a week.

The Very Long Baseline Array $\left(\mathrm{VLBA}^{14}\right)$ is an interferometer consisting of ten identical $25 \mathrm{~m}$ antennas on transcontinental baselines up to $8000 \mathrm{~km}$, which are remotely controlled from the Science Operations Center in Socorro, New Mexico. The received signals are amplified, digitized, and recorded on fast, high-capacity recorders and are sent from the individual VLBA stations to the correlator in Socorro. Observations are performed at frequencies from $1.2 \mathrm{GHz}$ to $96 \mathrm{GHz}$ in eight discrete bands and two narrow sub-GHz bands, including the primary spectral lines that produce high-brightness maser emission.

1ES 1011+496 has been monitored with the VLBA in MOJAVE at $15 \mathrm{GHz}$ since May 2009. MOJAVE ${ }^{15}$ is a long-term program that monitors radio brightness and polarization variations in jets associated with active galaxies visible in the northern sky (Lister et al. 2009). Seven observations have been performed on 1ES 1011+496 with the $2 \mathrm{~cm}$ VLBA from 2009 May

\footnotetext{
14 http://www.vlba.nrao.edu/

15 http://WWW. physics. purdue . edu/astro/MOJAVE/
}

to 2012 December with a cadence of one to two measurements per year.

\section{Results}

\subsection{MAGIC data}

After applying event selection cuts, the stacked analysis from both years yields an excess of $1002 \gamma$-like events above $100 \mathrm{GeV}$ within $0.026 \mathrm{deg}^{2}$ of the distribution of the squared angular distance $\theta^{2}$ between the reconstructed event direction and the catalog position of 1ES 1011+496. The background level of 5242 events was estimated by applying the same event cuts and using the anti-source position located at $180^{\circ}$ with respect to the reconstructed position of the source in the camera as Off region. We find a strong signal of $\sim 9.4 \sigma$ significance, calculated according to Li \& Ma (1983, Eq. (17)).

The daily VHE $\gamma$-ray light curve above $150 \mathrm{GeV}$ from 2011 and 2012 MAGIC observations is shown in Fig. 1. The fit of the light curve with a constant function gives a probability of $\sim 21 \%\left(\chi^{2} /\right.$ d.o.f. $\left.{ }^{16}=42 / 36\right)$ for non-variable emission at a mean flux level of $(1.46 \pm 0.16) \times 10^{-11} \mathrm{ph} \mathrm{cm}^{-2} \mathrm{~s}^{-1}$ corresponding to $(4.53 \pm 0.50) \%$ of the Crab Nebula flux (C.U.). During the $2011 / 2012$ observations, the integral flux above $200 \mathrm{GeV}$ is lower than the flux measured by MAGIC during the source discovery epoch in VHE $\gamma$ rays (Albert et al. 2007a) and the MWL campaign in 2008 (Ahnen et al. 2015), when the source was in a high state in this energy range (Table 1).

The differential spectrum (Fig. 2) shows good agreement with a simple power law in the range from $\sim 100 \mathrm{GeV}$ to $\sim 900 \mathrm{GeV}$. The flux normalization $f_{0}$ at $200 \mathrm{GeV}$ is equal to $(1.33 \pm 0.06) \times 10^{-10} \mathrm{ph} \mathrm{cm}^{-2} \mathrm{~s}^{-1} \mathrm{TeV}^{-1}$, and the photon index $\Gamma$ was found to be $3.66 \pm 0.22$. The spectrum was unfolded using the Tikhonov algorithm to correct for the finite energy resolution. Different unfolding algorithms as described in Albert et al. (2007b) were compared and found to agree within the errors. The systematic uncertainties in the spectral measurements with MAGIC stereo observations are $11 \%$ in the normalization factor (at $300 \mathrm{GeV}$ ) and $0.15-0.20$ in the photon index. The error on the flux does not include the uncertainty on the energy scale. The energy scale of the MAGIC telescopes is determined with a precision of about $17 \%$ at low energies $(E<100 \mathrm{GeV})$ and $15 \%$ at medium energies $(E>300 \mathrm{GeV})$. Further details are reported in Aleksić et al. (2012). The observed $\gamma$-ray flux was corrected for absorption by extragalactic background light (EBL) according to the model of Domínguez et al. (2011). The deabsorbed differential spectrum is in good agreement with a simple power law $\left(\chi^{2} /\right.$ d.o.f. $=2 / 4,69 \%$ fit probability $)$, which is parametrized

16 d.o.f.: Degrees of freedom. 


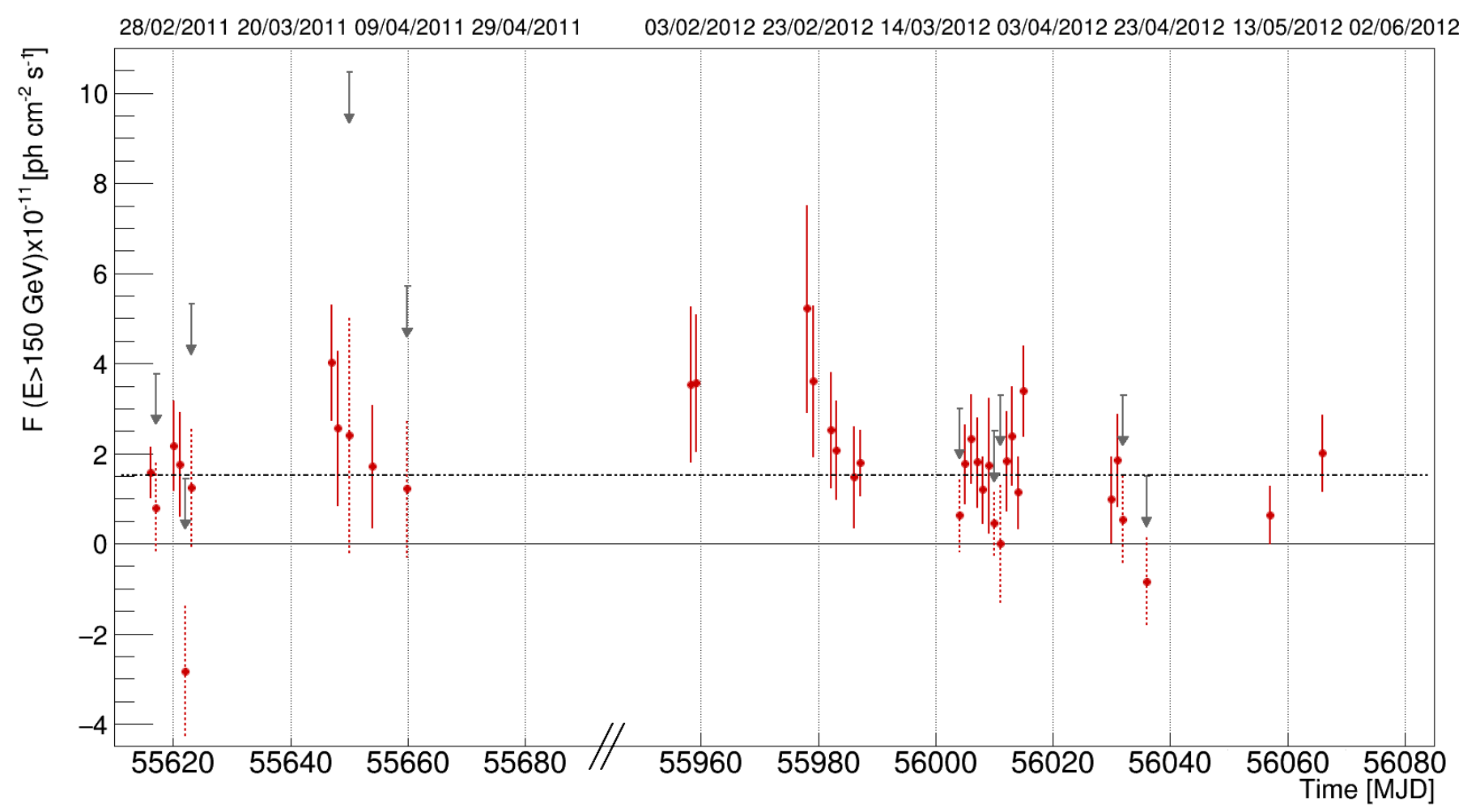

Fig. 1. Daily binned light curve of the integral VHE-ray emission (red points) from 1ES 1011+496 above 150 GeV during observations carried out from 2011 to 2012. Upper limits (gray arrows) at 95\% confidence level were derived according to the Rolke et al. (2005) method for each time bin where the observed integral flux was negative or with a flux estimation smaller than its error (red points with dashed error bars). The mean flux level (black dashed line) is retrieved from a fit with a constant to the light curve including the points that are negative or whose relative error is greater than $100 \%$.

by a photon index $\Gamma=3.0 \pm 0.3$ and a flux normalization $f_{0}$ at $200 \mathrm{GeV}$ of $(1.87 \pm 0.08) \times 10^{-10} \mathrm{ph} \mathrm{cm}^{-2} \mathrm{TeV}^{-1}$.

The energy range of the differential spectrum presented here is slightly extended to lower energies with respect to previous MAGIC observations (Table 1). The measurement of the spectral index is consistent with previous observations within the errors. The results on the normalized differential flux are consistent within the systematic errors and the intrinsic spectral slopes from a simple power law fit to the deabsorbed spectra show consistency within the statistical errors.

\subsection{Multiwavelength light curves}

In Fig. 3 the stitched 2011 and 2012 MWL light curves are presented. Moreover, we report the long-term behavior of the source (Fig. 4). The intrinsic variability amplitude was quantified with the fractional variability $F_{\text {var }}$ as defined in Vaughan et al. (2003). The uncertainty in $F_{\text {var }}$ was computed following the prescription from Poutanen et al. (2008) as described in Aleksić et al. (2015b). The fractional variability at different energies is reported in Fig. 5 for both the MAGIC 2011/2012 observations and the long-term datasets. The figure only shows those bands with positive excess variance (i.e., variance larger than the mean squared errors) because the fractional variability is not defined for negative excess variances. Such negative excess variances are interpreted as absence of variability, either because there was no variability or because the instruments were not sensitive enough to detect it.

Possible variations in the source emission in HE $\gamma$ rays shown in Fig. 3 have been tested following the same likelihood method described in the 2FGL catalogue (Nolan et al. 2012).

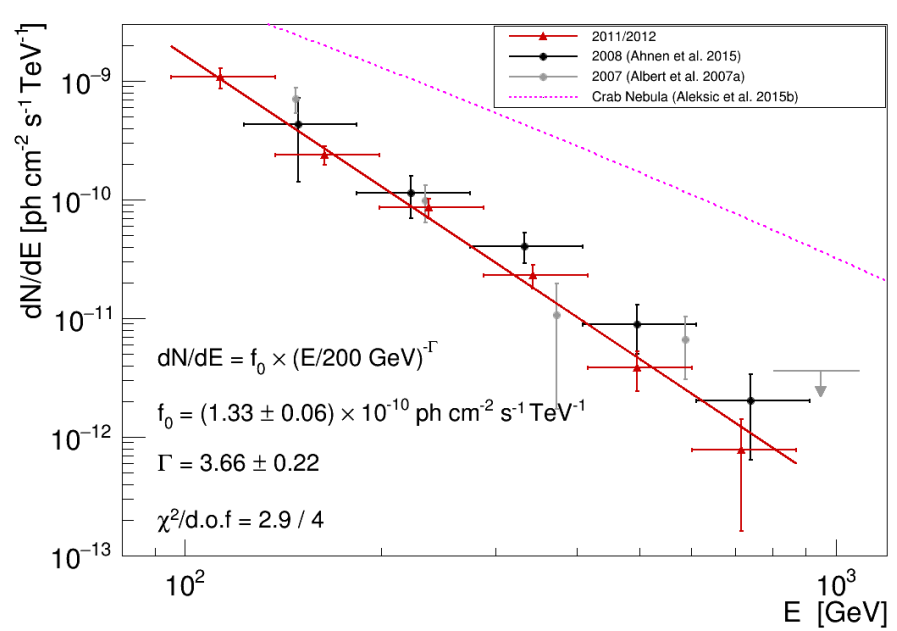

Fig. 2. Observed (red filled triangles) VHE $\gamma$-ray differential spectrum of 1ES 1011+496 from 2011 and 2012 MAGIC stereo data. The spectrum is fitted by a simple power law (red solid line) whose parameters are indicated in the inlet. For comparison, the differential spectra (gray and black circles) from mono observations in 2007 (Albert et al. 2007a) and 2008 (Ahnen et al. 2015) and the Crab Nebula spectrum (pink dashed line) are also plotted (Aleksić et al. 2015a).

The method, applied to the 2012 three-day binned light curve indicates that the flux is not significantly variable $\left(\mathrm{TS}_{\mathrm{var}}=\right.$ 48 for 49 d.o.f. $)^{17}$. For the 2011 and 2012 data samples, the time-averaged integrated flux in the Fermi-LAT energy 17 If the null hypothesis is correct, i.e., the source flux is constant across
the considered interval, $\operatorname{TS}_{\text {var }}$ is distributed as $\chi^{2}$ with 49 degrees of 


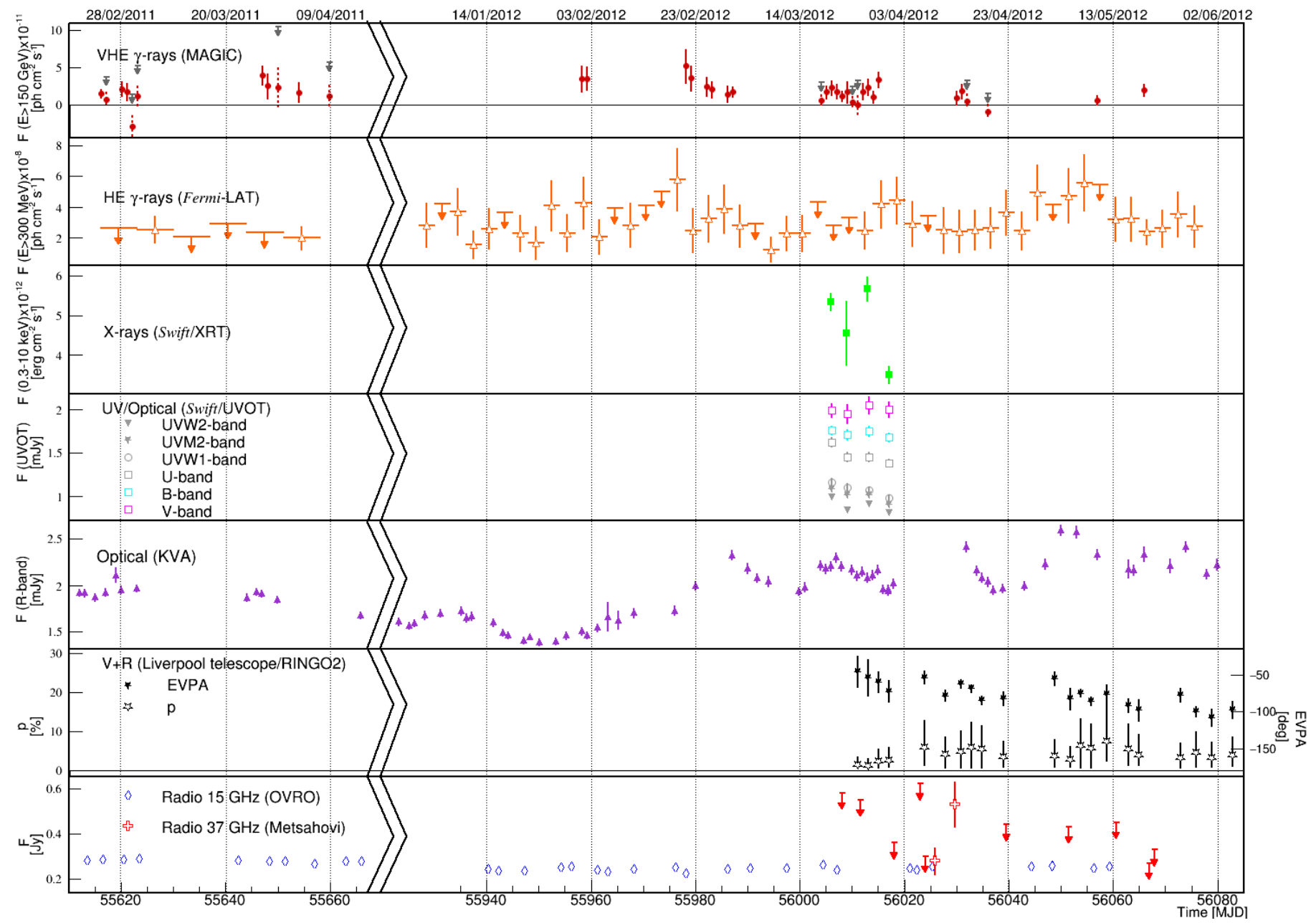

Fig. 3. Stitched 2011 and 2012 MWL light curve of 1ES 1011+496 zoomed into the observation periods from February to April and from January to May. From top to bottom: VHE $\gamma$-ray (red circles) and HE $\gamma$-ray (orange triangles) data by MAGIC and by Fermi-LAT, observations in X-rays (green squares), UV (gray triangles, stars and circles) and optical $U, B$ and $V$-bands (gray, cyan and magenta squares) by $S w i f t$ (XRT and UVOT), in the optical $R$-band (purple triangles) by the KVA telescope (host galaxy subtracted; Nilsson et al. 2007), optical polarimetry data taken with $V+R$ filter by the Liverpool telescope (RINGO2) and radio data provided by the OVRO (blue diamonds) and the Metsähovi telescopes (red crosses). Upper limits of $95 \%$ confidence level are indicated by downward arrows (see text for details). The light curves are daily binned except HE $\gamma$-rays, where a seven and three day binning was applied to the 2011 and 2012 data, respectively. The time axis between 2011 and 2012 observation is discontinuous.

range calculated from $300 \mathrm{MeV}$ to $300 \mathrm{GeV}$ is $(2.4 \pm 0.2) \times$ $10^{-8} \mathrm{ph} \mathrm{cm}^{-2} \mathrm{~s}^{-1}$ with a spectral index of $1.78 \pm 0.05(\mathrm{TS}=966)$.

The Swift/XRT data indicate some variability $\left(F_{\mathrm{var}}=0.18 \pm\right.$ $0.05)$ in X-rays $(0.3-10 \mathrm{keV})$, with a mean flux determined with a fit to the data points using a constant of $(4.7 \pm 0.1) \times$ $10^{-12} \mathrm{erg} \mathrm{cm}^{-2} \mathrm{~s}^{-1}$. The spectral indices obtained from a simple power-law fit to the data (Table 2) are in agreement within the errors. The optical and UV bands measured with Swift/UVOT show a very modest variability $(<\sim 8 \%)$ in comparison with fractional variability measured in the $R$-band $(13 \%)$. This relatively low variability measured with $S$ wift/UVOT could be related to the very limited temporal coverage during the coordinated multiinstrument observations in 2011/2012 (see Fig. 3). Previous observations of this object showed a higher $R$-band flux (see Albert et al. 2007a; Ahnen et al. 2015) and the fractional variability of the long-term light curve in this band exceeds $25 \%$.

The radio emission monitored by the Metsähovi $(37 \mathrm{GHz})$ and OVRO $(15 \mathrm{GHz})$ telescopes shows variability in both cases

freedom, and a value of $\mathrm{TS}_{\mathrm{var}}>74.9$ is used to identify variable sources at a $99 \%$ confidence level.
$\left(F_{\text {var }}=0.39 \pm 0.13\right.$ and $0.061 \pm 0.006$, respectively $)$ with mean flux levels of $(0.35 \pm 0.05) \mathrm{Jy}$ and $(0.196 \pm 0.027) \mathrm{Jy}$ and a change in flux of $0.23 \mathrm{Jy}(77 \%)$ and $0.06 \mathrm{Jy}(28 \%)$, respectively. Given the small statistical errors associated with observations at 15 $\mathrm{GHz}$, the mean flux level of $(0.246 \pm 0.001) \mathrm{Jy}$ appears slightly lower in 2012 compared to $(0.277 \pm 0.001) \mathrm{Jy}$ in 2011 . In the case of the OVRO data, the variability was also studied in Richards et al. (2014), who calculated the intrinsic modulation index using four years of OVRO data between 2008 and 2012. The intrinsic modulation index (defined as intrinsic standard deviation over intrinsic mean flux density) describes the variability of the source when sampling effects and observational uncertainties are accounted for (Richards et al. 2011). The intrinsic modulation index for $1 \mathrm{ES} 1011+496$ is $(0.054 \pm 0.004) \mathrm{Jy}$, corresponding to a variability amplitude of $5 \%$ indicating modest variability.

The comparison of the long-term radio light curves compiled from OVRO (Fig. 4) and MOJAVE observations indicates that the decreasing trend of the flux observed by OVRO most likely originates from the radio core (blank black circles), which follows this trend, while the flux emission of the jet components 


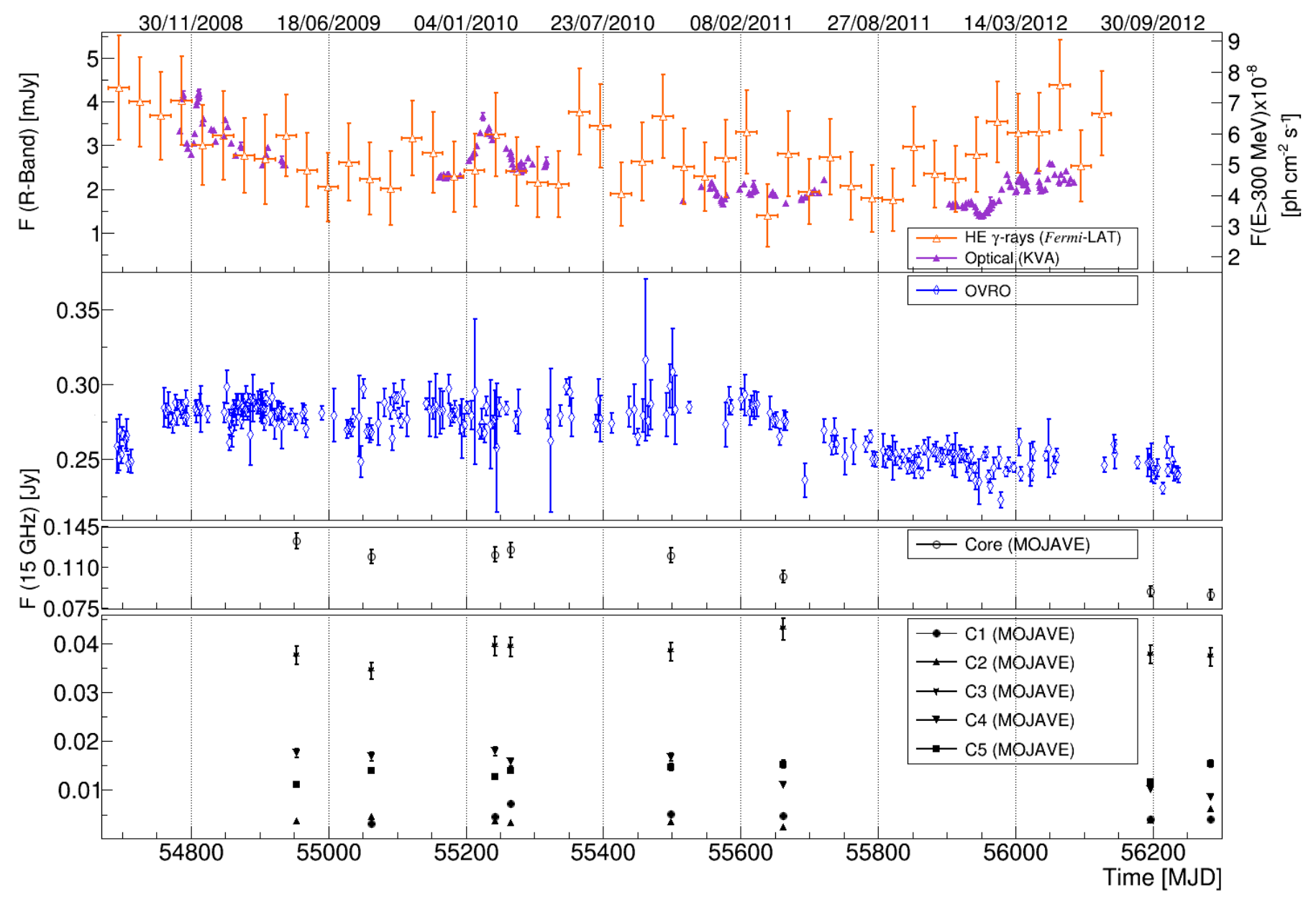

Fig. 4. Long-term MWL light curves of 1ES 1011+496. In the top panel the monthly binned HE $\gamma$-ray light curve (orange triangles) from the 3FGL (Acero et al. 2015) and daily binned optical $R$-band light curve (purple triangles; host galaxy subtracted; Nilsson et al. 2007) from the KVA telescope are shown. The radio data at $15 \mathrm{GHz}$ of the OVRO telescope (blue diamonds) and MOJAVE (black markers) are reported in the lower panels. MOJAVE provides flux measurements of the radio core (open circles) and the various jet components (C1 to C5; filled symbols).
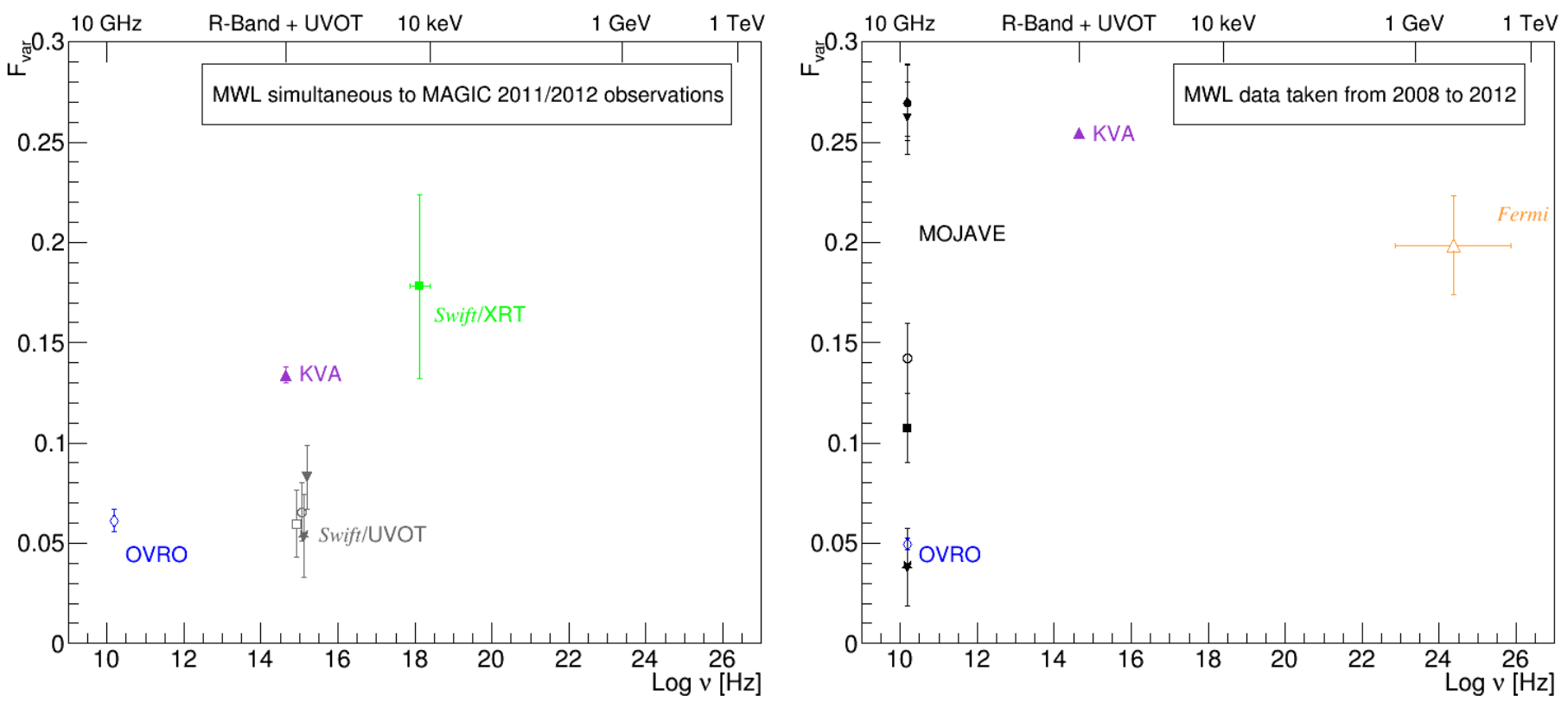

Fig. 5. Fractional variability amplitude, $F_{\text {var }}$ as a function of frequency for data simultaneous to the MAGIC observation periods (left) shown in Fig. 3 and long-term data samples (right) shown in Fig. 4. The $F_{\text {var }}$ of the radio core (right) is indicated by an open circle, while the values computed for the components $\mathrm{C} 1$ to $\mathrm{C} 5$ are represented by filled symbols (C1: circle; $\mathrm{C} 2$ : upward triangle; $\mathrm{C} 3$ : star; $\mathrm{C} 4$ : downward triangle; C5: square). 
Table 2. Log and fitting results of Swift/XRT observations of 1 ES $1011+496$ in the $0.3-10 \mathrm{keV}$ band using a power-law model with $N_{\mathrm{H}}$ fixed to Galactic absorption.

\begin{tabular}{ccccc}
\hline \hline $\begin{array}{c}\text { Observation } \\
\text { Date }\end{array}$ & $\begin{array}{c}\text { Net exposure time } \\
\mathrm{s}\end{array}$ & $\begin{array}{c}\text { Photon index } \\
\Gamma\end{array}$ & $\begin{array}{c}\text { Flux } 0.3-10 \mathrm{keV}^{a} \\
{\left[10^{-13} \mathrm{erg} \mathrm{cm}^{-2} \mathrm{~s}^{-1}\right]}\end{array}$ & $\begin{array}{c}\chi^{2} / \\
\text { d.o.f. }\end{array}$ \\
\hline 2012 Mar. 20 & 2285 & $2.35 \pm 0.06$ & $5.34 \pm 0.22$ & $56 / 62$ \\
2012 Mar. 23 & 210 & $2.50 \pm 0.26$ & $4.55 \pm 0.81$ & $\mathrm{Cash}^{b}$ \\
2012 Mar. 27 & 1618 & $2.12 \pm 0.08$ & $5.67 \pm 0.31$ & $46 / 39$ \\
2012 Mar. 31 & 2195 & $2.33 \pm 0.08$ & $3.50 \pm 0.22$ & $39 / 35$ \\
\hline
\end{tabular}

Notes. ${ }^{(a)}$ Observed flux; ${ }^{(b)}$ the Cash statistic (Humphrey et al. 2009) was used to fit the spectrum.

seems to vary randomly (filled black symbols). Thus, variability in the radio flux can most likely be associated with the radio core. However, the fractional variability amplitude values for the various jet components indicate that the variability observed in radio could also be associated with the radio jet.

\subsection{Long-term correlation studies}

We studied the correlations between the light curves in radio, optical $R$-band, and HE $\gamma$ rays reported in Fig. 4. For the radio/optical correlation we used only observations for which the difference in observation time was less than one day, resulting in a sample of 56 data points. Since the HE $\gamma$-ray light curve $^{18}$ is binned monthly, we rebinned the radio and optical data using the HE $\gamma$-ray light curve bin edges to match the data samples, providing a sample of 45 and 26 points in the case of radio/HE gamma-ray and optical/HE gamma-ray correlation, respectively.

Although the optical light curve seems to show many features that are uncorrelated to simultaneous radio observations, we find a significant $(5.4 \sigma)$ linear (Pearson) correlation of $0.63_{-0.09}^{+0.08}$ strength between radio and optical, which is driven by the decrease in the radio and optical flux around MJD 55700. No significant linear correlation was found between the optical band and HE $\gamma$ rays and radio frequencies and HE $\gamma$ rays.

\subsection{Optical and radio polarimetry}

The optical polarimetry data display a very low degree of optical polarization $(P)$ with a mean value of $2.5 \pm 0.6 \%$ (Fig. 3 ). The epochs of optical polarimetry measurements coincide with those when the photometric KVA data exhibit smooth low-amplitude oscillations in the total flux, but no correlation is observed. In fact, no significant variability is detected in $P$ and the statistical errors of the low-level polarization measurements are dominating. The electric vector position angle (EVPA) shows a general trend throughout the observation period by which the angle steadily decreases from roughly $-50^{\circ}$ to about $-100^{\circ}$.

Figure 6 shows the multi-epoch $15 \mathrm{GHz}$ radio map of the source provided by MOJAVE. The radio morphology consists of a compact optically thick core, and more diffuse jet emission that extends to the west. In the observed dates, from May 2009 to December 2012, the jet position angle (PA) is stable, oriented at $-100^{\circ}$ to $-80^{\circ}$, approximately. This is compatible with previous measurements by Augusto et al. (1998) and Nakagawa et al. (2005), who reported values of $-99^{\circ}$ and $-105^{\circ}$, respectively, at this frequency.

\footnotetext{
${ }^{18}$ Data taken from the 3FGL (Acero et al. 2015), available at http: //heasarc.gsfc.nasa.gov/W3Browse/fermi/fermilpsc.html
}

Table 3. EVPA and mean fractional linear polarization of the radio core and jet at $15 \mathrm{GHz}$ from seven epochs of MOJAVE observations.

\begin{tabular}{ccccc}
\hline \hline $\begin{array}{c}\text { Observation } \\
\text { Date }\end{array}$ & $\begin{array}{c}\mathrm{EVPA}_{\text {Core }} \\
{\left[{ }^{\circ}\right]}\end{array}$ & $\begin{array}{c}\mathrm{EVPA}_{\text {Jet }} \\
{\left[{ }^{\circ}\right]}\end{array}$ & $\begin{array}{c}p_{\text {Core }} \\
{[\%]}\end{array}$ & $\begin{array}{c}p_{\text {Jet }} \\
{[\%]}\end{array}$ \\
\hline 2009 May 02 & -46 & -25 & 1 & 6 \\
2009 Aug. 19 & -37 & -29 & 3 & 12 \\
2010 Mar. 10 & -19 & -25 & 2 & 11 \\
2010 Sept. 29 & -15 & -24 & 2 & 18 \\
2011 Apr. 11 & -163 & -16 & 1 & 11 \\
2012 Sept. 27 & -126 & -41 & 3 & 8 \\
2012 Dec. 23 & -123 & -27 & 2 & 7 \\
\hline
\end{tabular}

Notes. The EVPA accuracy is roughly $\pm 5^{\circ}$. For the jet the values of the fractional linear polarization are averaged over the whole jet excluding the core. Thus localized regions in Fig. 6 have higher and lower fractional polarization values than those listed here.

The EVPA in radio behaves differently at earlier and later epochs: before 2011, the core EVPA is decreasing from $\sim-45^{\circ}$ to $-15^{\circ}$, whereas the jet EVPA is rather stable at roughly $-25^{\circ}$ (Table 3). In 2011 the core EVPA is about $-160^{\circ}$, the electric vector having moved in the clockwise direction from its original position to a final angle of roughly $-120^{\circ}$; the jet EVPA remained constant during all epochs, at about $-15^{\circ}$ to $-40^{\circ}$.

The most interesting feature of the jet polarization in radio is a relatively large activity in the amount of fractional linear polarization seen, with some bright features appearing at different times and positions within the jet that have a degree of fractional linear polarization up to $60 \%$, close to the maximum value expected from homogeneous synchrotron sources (Pacholczyk 1970). These values of fractional linear polarization are much higher than what is seen in the optical, and in fact appear to bear little resemblance to the general state of the source polarization at these higher frequencies. The values of the fractional linear polarization reported in Table 3 are averaged over the whole jet excluding the core. Thus, localized regions in Fig. 6 have both higher and lower fractional polarization values.

The relation between the optical and radio EVPA is further complicated by the fact that the optical EVPA follows a counterclockwise rotation trend throughout the year 2012, when optical polarization data was taken, going from $150^{\circ}$ to $100^{\circ}$ (or equivalently $-30^{\circ}$ to $-80^{\circ}$ if we allow for the $180^{\circ}$ ambiguity in the EVPA definition). This trend is opposite to that followed by the radio core EVPA in 2012 and therefore appears to dissociate the optical polarized emission, or at least the bulk of it, from what is happening at the radio core. But when we look at the jet EVPA in radio an agreement is found with the behavior seen in optical. According to Table 3, in the last two epochs of radio data, the overall radio jet EVPA was pointing between $-15^{\circ}$ and 


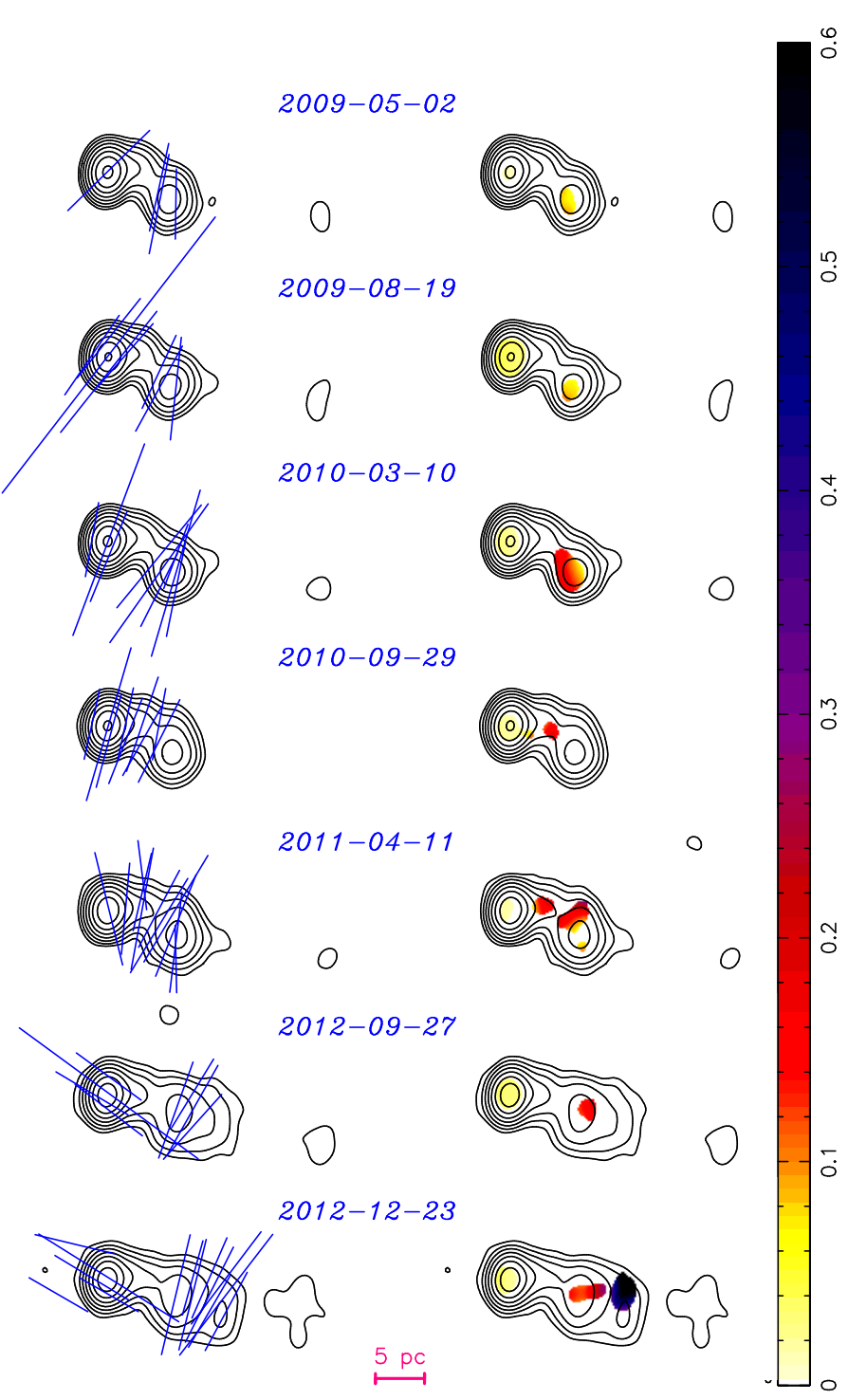

Fig. 6. MOJAVE $15 \mathrm{GHz}$ VLBA images of 1ES 1011+496 at seven epochs from 2009 to 2012. The left-hand images show total intensity contours with electric polarization vectors overlaid in blue. The righthand images show total intensity contours with fractional linear polarization in color ranging from 0 to 0.6 . The images have been convolved with the same Gaussian restoring beam having dimensions $0.83 \times$ 0.63 mas and position angle $-5^{\circ}$. In all images, the contour levels are factor of 2 multiples of the base contour level of $0.9 \mathrm{mJy}_{\text {beam }}{ }^{-1}$. The polarization vectors have a scaling of $2 \mathrm{mJy} \mathrm{beam}^{-1} \mathrm{mas}^{-1}$ and are indicated for regions with polarized flux density exceeding $0.8 \mathrm{mJy}_{\text {beam }}{ }^{-1}$. The angular scale of the images is $3.4 \mathrm{pc} \mathrm{mas}^{-1}$.

$-40^{\circ}$. The direction is off by quite a few degrees, but it is similar to what is established in optical. Furthermore the trend is also counter-clockwise.

Although optical and radio jet polarized emission cannot be confidently associated on this basis alone, one has to keep in mind that from the radio maps, the jet structure is quite complex with bright features characterized by quite highly polarized emission levels. Likewise, the polarization vectors that are associated with these individual regions do not all behave the same or have the same orientations. Based on that we could speculate that one or more of these bright features seen in radio are also the zones responsible for the bulk of the optical polarized emission - as would be logical to expect - but in optical, differently from radio, the absence of good-enough spatial resolution prevents one from getting a clear picture. In fact, the poor spatial resolution would have the effect of lowering the net polarization of the source, as regions with slightly different polarization directions are seen superposed and the net effect of a preferential direction of the field is washed away. Nevertheless, the fact that we see a broad orientation for the optical EVPA towards the same rough direction of the radio jet EVPA, and that the trend of rotation of both also matches, can be taken as an indication that the optical emission is also produced in the bright features of the jet. If these are zones of particle acceleration, for example shocked plasma zones where the field intensity and degree of ordering is also enhanced, then this would provide provide some insight on the nevertheless complex dynamics of the source.

\subsection{Jet kinematics}

Based on the first five epochs presented in Fig. 6, a statistically significant $(\geq 3 \sigma)$ expansion rate of $131 \pm 27 \mu \mathrm{as} \mathrm{yr}^{-1}$ corresponding to an apparent speed of $1.8 \pm 0.4 c$ was found for the bright jet feature at 2 mas from the core (Lister et al. 2013). The last epoch has poor data quality due to three VLBA antenna drop-outs. No other components display motion at such statistical significance. Out of the 45 known TeV HBLs ${ }^{19}$, 13 have been targets of VLBA measurements (Lister et al. 2013; Piner \& Edwards 2013; Tiet et al. 2012; Piner et al. 2010). The majority of these HBLs show rather low apparent speeds, i.e., $<1 c$. In addition to 1ES 1011+496, a superluminal motion (e.g., Urry \& Padovani 1995; Ghisellini 2000) of 1.2 $\pm 0.4 c$ (Piner et al. 2010) was measured for the HBL H $1426+428$ with a statistical significance of $\geq 2 \sigma$. Given the statistical error, the apparent speed of this motion could also be $<1 c$, which makes 1ES 1011+496 the HBL with the highest statistically significant superluminal speed measured so far. However, since the measured apparent speed for this source is still compatible with the speed of light within $2 \sigma$, a highly significant detection of superluminal motion in a $\mathrm{TeV}$ HBL cannot be claimed yet.

\section{Modeling the SED}

Owing to the general low state of the source in the observed energy bands in 2011 and 2012, the data were combined to an average SED (Fig. 7), except X-ray observations, where the highest (2012 March 27) and lowest (2012 March 31) flux observed are reported instead. Corrections for EBL absorption were applied to the VHE $\gamma$-ray data according to the model by Domínguez et al. (2011), while the data from Swift in the UV bands and optical data in the $R$-band from the KVA telescope were corrected for Galactic extinction (Fitzpatrick 1999) and host galaxy contribution (Nilsson et al. 2007), respectively. For comparison, we show archival data available at the ASI Science Data Center $(\mathrm{ASDC})^{20}$. Both the low- and high-energy bump of the SED are well constrained by these simultaneous MWL data. For the latter, a connection of the VHE and HE $\gamma$-ray band was achieved for the first time for 1ES 1011+496. The SSC model used to describe the data locates the peak of the inverse Compton bump at around $20 \mathrm{GeV}$.

The SED shows no indication for the previous hypothesis of an inverse Compton dominance (Albert et al. 2007a). This previous assumption is likely related to missing complementary MWL data, whereby both peaks were barely constrained.

${ }^{19}$ http://tevcat . uchicago . edu; current catalog version: 3.400 . 
Table 4. Input model parameters assumed for the SSC model (Maraschi \& Tavecchio 2003) shown in Fig. 7.

\begin{tabular}{cccccccccc}
\hline \hline Year & $\begin{array}{c}\gamma_{\min } \\
{\left[10^{3}\right]}\end{array}$ & $\begin{array}{c}\gamma_{\mathrm{b}} \\
{\left[10^{4}\right]}\end{array}$ & $\begin{array}{c}\gamma_{\max } \\
{\left[10^{5}\right]}\end{array}$ & $n_{1}$ & $n_{2}$ & $\begin{array}{c}B \\
{[\mathrm{G}]}\end{array}$ & $\begin{array}{c}K \\
{\left[10^{3} \mathrm{~cm}^{-3}\right]}\end{array}$ & $\begin{array}{c}R \\
{\left[10^{16} \mathrm{~cm}\right]}\end{array}$ & $\delta$ \\
\hline $2007^{a}$ & 3.0 & 5.0 & 200 & 2.0 & 5.0 & 0.15 & 20 & 1.0 & 20 \\
$2008^{b}$ & 7.0 & 3.4 & 8.0 & 1.9 & $3.3(3.5)$ & 0.048 & $0.7(0.8)$ & 3.25 & 26 \\
$2011 / 2012^{I}$ & 10.0 & 4.0 & 7.0 & 2.0 & 3.7 & 0.19 & 10.0 & 1.0 & 20 \\
$2011 / 2012^{I I}$ & 10.0 & 3.3 & 4.0 & 2.0 & 3.8 & 0.19 & 13.4 & 0.9 & 20 \\
\hline
\end{tabular}

Notes. The parameters for the SED modeling of previous observations are shown for comparison. ${ }^{(I)}$ X-ray spectrum from March 27 ; ${ }^{(I I)} \mathrm{X}$-ray spectrum from March 31; ${ }^{(a)}$ Albert et al. (2007a); ${ }^{(b)}$ Ahnen et al. (2015). The parameters reported for the modeling of the 2008 data consider the high (low) state observed in X-rays, while those listed for the modeling of the 2007 data are based on the MAGIC spectrum that has been corrected for EBL absorption using the model by Kneiske et al. (2002) current at that time.

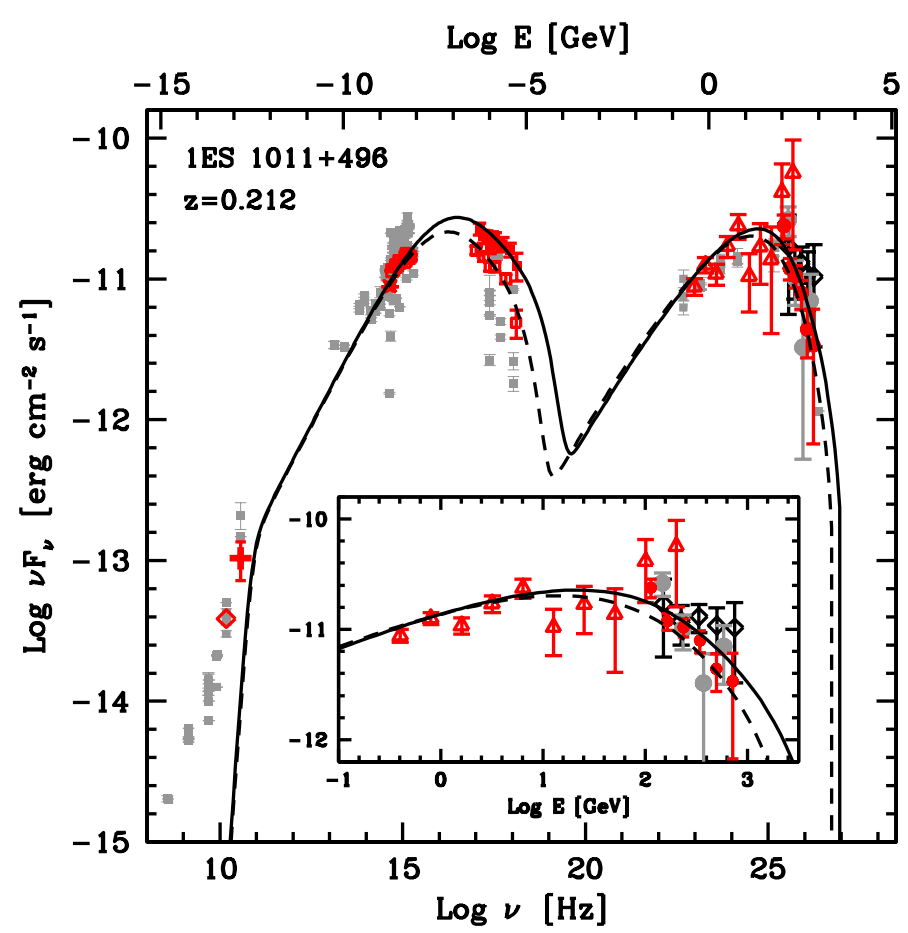

Fig. 7. Averaged SED of 1ES 1011+496 compiled from simultaneous 2011 and 2012 MWL observations marked in red. We combine deabsorbed (Domínguez et al. 2011) VHE $\gamma$-ray observations (circles) by MAGIC and HE $\gamma$-ray data (triangles) from Fermi-LAT; Swift data from 2012 March 27 (filled squares) and 31 (open squares) in X-rays and UVOT bands (squares), the latter corrected for Galactic extinction (Fitzpatrick 1999); optical data in the $R$-band (star) from KVA (corrected for host galaxy contribution; Nilsson et al. 2007) and radio data at $15 \mathrm{GHz}$ (diamond) and $37 \mathrm{GHz}$ (cross) provided by the OVRO and Metsähovi telescopes. The solid (dashed) line represents the fit with a one-zone SSC model considering the X-ray spectrum from March 27 (March 31). The parameters are listed in Table 4. Previous MAGIC observations carried out in 2007 (black diamonds; Albert et al. 2007a) and 2008 (gray circles; Ahnen et al. 2015) are corrected for EBL absorption according to the model by Domínguez et al. (2011). The inset is a zoom into the HE to VHE $\gamma$-ray band. Archival data (gray squares) are taken from the $\mathrm{ASDC}^{20}$.

From the SED presented here, the maximum flux $v F_{v}$ of both energy bumps seems to be nearly equal $\left(2.75 \times 10^{-11} \mathrm{erg} \mathrm{cm}^{-2} \mathrm{~s}^{-1}\right.$ and $2.26 \times 10^{-11} \mathrm{erg} \mathrm{cm}^{-2} \mathrm{~s}^{-1}$ for the synchrotron and inverse Compton peak, respectively).

A one-zone SSC model (Maraschi \& Tavecchio 2003) was applied to reproduce the broadband SED, assuming a spherical emission region of radius $R$ filled with a tangled magnetic field strength $B$. A primary spectrum of a relativistic electron population is approximated by a smoothed, broken power law that is parametrized by the minimum $\left(\gamma_{\min }\right)$, break $\left(\gamma_{\mathrm{b}}\right)$, and maximum $\left(\gamma_{\max }\right)$ Lorentz factors; the slopes before $\left(n_{1}\right)$ and after $\left(n_{2}\right)$ the break; and the electron density parameter $K$. Relativistic effects are taken into account by the Doppler factor $\delta$. Absorption of $\gamma$ rays in the emitting region by photon-photon pair production on internal soft (e.g., synchrotron) photons (e.g., Dondi \& Ghisellini 1995) is self-consistently accounted for in the model, but negligible $(\tau \ll 1)$ for the current set of parameters. The emission is self-absorbed at radio frequencies, implying that it is dominated by the outer regions of the jet. Therefore, radio data are not included in the SED modeling. However, the predicted radio flux of the emission region does not violate the observed value, showing variations over half-year long timescales (Fig. 4), which hint to emission regions that are likely associated with scales larger than those commonly considered for the highenergy emission in sources of this kind. The parameters of the one-zone SSC model can be uniquely fixed once the SED peaks (frequencies and luminosities) and the variability timescales are known (Tavecchio et al. 1998). The physical parameters assumed for this model are listed in Table 4 together with those derived from 2007 and 2008 observations using the same model. In the present case we do not have any estimate of the variability timescale, which is directly linked to the source size, and thus the set of parameters cannot be fully constrained. We thus assume a radius of the emitting region and a Doppler factor close to $R \approx 10^{16} \mathrm{~cm}$ and $\delta=20$, values commonly found in sources of this kind (e.g., Tavecchio et al. 2010; Aleksić et al. 2014b, $2015 c, d)$. The other parameters derived by reproducing the SED are also similar to those typically inferred for HBLs (Tavecchio et al. 2010). In particular the low magnetic field strength is quite common for HBLs (e.g., Finke et al. 2008; Dermer et al. 2015) rather than being typical for IBLs, leading to deviations from equipartition.

The cooling time for the electrons emitting at the synchrotron peak (considering both synchrotron and inverse Compton losses) $t_{\text {cool }}$ is $2.7 \times 10^{5} \mathrm{~s}$, which is quite close to the escape time of $t_{\mathrm{esc}} \sim R / c=3 \times 10^{5} \mathrm{~s}$ suggested by Tavecchio et al. (1998). The energy density of the electrons and the magnetic field $U_{\mathrm{e}}$ and $U_{B}$ correspond to $7.3 \times 10^{-2}$ and $1.4 \times 10^{-3} \mathrm{erg} \mathrm{cm}^{-3}$ indicating that the magnetic field is far below equipartition, $U_{B} / U_{\mathrm{e}}=0.02$. A quite general result in the framework of the one-zone SSC model for TeV emitting BL Lacs is the high ratio of $U_{\mathrm{e}} / U_{\mathrm{B}}$, indicating that the particle energy density is largely dominating over the magnetic energy density. This is quite a robust result and represents a problem for both jet theory and the particle acceleration model (e.g., Tavecchio \& Ghisellini 2016, and references therein). Possible solutions include inhomogeneous models such 
as the so-called structured jet model. In this specific case, the jet is thought to be composed of a fast spine, which is responsible for the emission observed from blazars, surrounded by a slower sheath. The large photon energy density in the emitting region, provided by the sheath, allows the magnetic energy density to be increased in the spine, thus decreasing the $U_{\mathrm{e}} / U_{\mathrm{B}}$ ratio required to reproduce the observed SED.

As for other TeV HBLs (Piner \& Edwards 2013), the Lorentz factor derived from the modeling of the SED is larger than that inferred for the superluminal speed measured in the radio band. A possible explanation of the problem is that the jet decelerates from the innermost blazar region to the outer regions responsible for the radio emission (e.g., Georganopoulos \& Kazanas 2003), or that the radio and $\mathrm{TeV}$ emission derive from separate regions, the former being produced in a slow layer surrounding a fast, $\mathrm{TeV}$ emitting spine (Ghisellini et al. 2005).

The comparison with previous models of the source SED (Albert et al. 2007a; Ahnen et al. 2015) indicates a good agreement for most of the parameters. The radius of the emitting region derived in Ahnen et al. (2015) is about a factor of 3 larger than in the other cases. The minimum and maximum Lorentz factors show relatively large variations among the models but these parameters are usually not well constrained by the available data. The parameters from the 2008 modeling are in good agreement with the model presented here. Most likely, variations among the individual parameters are related to the previously poor MWL coverage rather than to important variations of the physical processes operating in 1ES 1011+496.

\section{Conclusion}

While the time-averaged VHE spectrum observed in 2011 and 2012 is consistent in spectral slope with MAGIC observations from 2007 and 2008, the integral flux above $200 \mathrm{GeV}$ is lower than previous VHE observation epochs. The deabsorbed VHE $\gamma$ ray spectrum, for which EBL corrections were applied, is in good agreement with a power law, with a spectral index that is consistent within the statistical errors with previous measurements of this parameter.

The MWL data of 1ES 1011+496 from 2011 and 2012 indicated a general low state of the source across the electromagnetic spectrum. We did not find statistically significant variability in VHE and HE $\gamma$ rays, while in the $R$-band the source varied notably without undergoing any major flare. The flux in the UV and $U$-bands showed a decreasing trend; however, owing to the small observation window in X-rays and the UVOT bands, no clear conclusion can be drawn on the variability in these wavebands. Low variability was found at $15 \mathrm{GHz}$, while a hint for moderate variability seemed to be on the signal at $37 \mathrm{GHz}$ that can most likely be associated with the radio core. Studies of the long-term light curves showed a significant linear connection between optical and radio indicating a correlated variability between these frequencies. The study of the optical and radio light curves with the HE $\gamma$-ray Fermi light curve did not show any significant linear correlation.

The source has been observed in optical and radio polarization at several epochs since 2009. VLBI data from 2009 to 2010 showed that the EVPA of the radio jet was constant, aligned at around $-25^{\circ}$, while the EVPA of the radio core decreased from about -45 to roughly $-15^{\circ}$. In 2011 the EVPA of the core underwent a rotation of nearly $100^{\circ}$ in the clockwise direction from its initial value, arriving at a final angle of about $-125^{\circ}$. In the jet, features with very high values of polarization of up to $60 \%$ were observed. These polarization features do not seem to contribute too much to the optical polarization emission, or are largely diluted by non-polarized emission, as the optical degree of polarization is very low $(<5 \%)$ and almost constant throughout the campaign. That said, a trend of slow counter-clockwise rotation was observed in the optical EVPA in 2012, in the same direction from certain components of the jet at the latest VLBI epochs. This similarly concurrent trend of EVPA rotation in optical and radio frequencies suggests that at least part of the optical emission has its origin in some of the bright radio features as detected by the VLBI observations. A contribution to the optical emission from other parts of the jet with different orientations of the magnetic field could also explain both the low level of polarization from the unresolved optical source and the non-exact alignment between any of the radio components and the optical EVPAs. In addition, we reported a detection of superluminal motion of $1.8 \pm 0.4 c$ in $1 \mathrm{ES} 1011+496$, which is the highest speed statistically significant $(\geq 3 \sigma)$ measured so far in a TeV HBL.

The one-zone SSC model was able to reproduce the broadband SED of 1ES 1011+496, which was derived from simultaneous 2011 and 2012 MWL data with parameters similar to those typically inferred for other HBL objects. From the SED presented here, the flux of both energy bumps seems to be nearly equal, being a typical HBL characteristic. The position of the synchrotron peak of the averaged 2011/2012 SED during the generally low emission state also favors an HBL nature of the source. The Lorentz factor derived from the modeling of the SED is larger than that inferred for the superluminal speed measured in the radio band, which can be explained by a deceleration of the jet from the innermost blazar region to the outer regions responsible for the radio emission. Another explanation could be that the radio and $\mathrm{TeV}$ emission originate from separate regions, where the former is produced in a slow layer surrounding a fast, TeV emitting spine. In general, the model parameters are in good agreement with those adopted for the SEDs from 2007 and 2008 MWL observations. Thanks to the connection of the VHE and HE energy band jointly observed for the first time for this source, the frequency of the IC peak was well constrained. The SSC model describing the SED located the peak of the inverse Compton bump at $\sim 20 \mathrm{GeV}$. In the VHE range, an extension to lower energies was reached in these new observations.

Acknowledgements. We would like to thank the Instituto de Astrofísica de Canarias for the excellent working conditions at the Observatorio del Roque de los Muchachos in La Palma. The financial support of the German BMBF and MPG, the Italian INFN and INAF, the Swiss National Fund SNF, the ERDF under the Spanish MINECO (FPA2012-39502), and the Japanese JSPS and MEXT is gratefully acknowledged. This work was also supported by the Centro de Excelencia Severo Ochoa SEV-2012-0234, CPAN CSD200700042, and MultiDark CSD2009-00064 projects of the Spanish ConsoliderIngenio 2010 programme, by grant 268740 of the Academy of Finland, by the Croatian Science Foundation (HrZZ) Project 09/176 and the University of Rijeka Project 13.12.1.3.02, by the DFG Collaborative Research Centers SFB823/C4 and SFB876/C3, and by the Polish MNiSzW grant 745/N-HESSMAGIC/2010/0. The Fermi-LAT Collaboration acknowledges generous ongoing support from a number of agencies and institutes that have supported both the development and the operation of the LAT as well as scientific data analysis. These include the National Aeronautics and Space Administration and the Department of Energy in the United States, the Commissariat à l'Énergie Atomique and the Centre National de la Recherche Scientifique/Institut National de Physique Nucléaire et de Physique des Particules in France, the Agenzia Spaziale Italiana and the Istituto Nazionale di Fisica Nucleare in Italy, the Ministry of Education, Culture, Sports, Science and Technology (MEXT), High Energy Accelerator Research Organization (KEK) and Japan Aerospace Exploration Agency (JAXA) in Japan, and the K. A. Wallenberg Foundation, the Swedish Research Council and the Swedish National Space Board in Sweden. Additional support for science analysis during the operations phase is gratefully acknowledged from the Istituto Nazionale di Astrofisica in Italy and the Centre National d'Études Spatiales in France. The Metsähovi team acknowledges the support from the Academy of Finland to our observing projects (Nos. 212656, 
210338,121148 , and others). The OVRO 40-m monitoring program is supported in part by NASA grants NNX08AW31G and NNX11A043G, and NSF grants AST-0808050 and AST-1109911. The National Radio Astronomy Observatory is a facility of the National Science Foundation operated under cooperative agreement by Associated Universities, Inc. This work made use of the Swinburne University of Technology software correlator (Deller et al. 2011), developed as part of the Australian Major National Research Facilities Programme and oper ated under licence. The MOJAVE project is supported under NASA-Fermi grant NNX12A087G. Part of this work is based on archival data provided by the ASI ASDC

\section{References}

Abdo, A. A., Ackermann, M., Ajello, M., et al. 2010a, ApJ, 716, 30 Abdo, A. A., Ackermann, M., Ajello, M., et al. 2010b, Nature, 463, 919 Acero, F., Ackermann, M., Ajello, M., et al. 2015, ApJS, 218, 23 Ackermann, M., Ajello, M., Albert, A., et al. 2012, ApJS, 203, 4 Ackermann, M., Ajello, M., Allafort, A., et al. 2013, ApJS, 209, 34 Aharonian, F., Akhperjanian, A., Beilicke, M., et al. 2004, A\&A, 421, 529 Ahnen, M. L., Ansoldi, S., Antonelli, L. A., et al. 2015, MNRAS, in press [arXiv: 1603.07308]

Albert, J., Aliu, E., Anderhub, H., et al. 2007a, ApJ, 667, L21

Albert, J., Aliu, E., Anderhub, H., et al. 2007b, NIMPA, 583, 494

Albert, J., Aliu, E., Anderhub, H., et al. 2008a, ApJ, 681, 944

Albert, J., Aliu, E., Anderhub, H., et al. 2008b, ApJ, 674, 1037

Albert, J., Aliu, E., Anderhub, H., et al. 2008c, NIPMA, 588, 424

Aleksić, J., Antonelli, L. A., Antoranz, P., et al. 2010, A\&A, 524, A77

Aleksić, J., Antonelli, L. A., Antoranz, P., et al. 2011, ApJ, 729, 115

Aleksić, J., Alvarez, E. A., Antonelli, L. A., et al. 2012, Astropart. Phys., 35, 435

Aleksić, J., Ansoldi, S., Antonelli, L. A., et al. 2014a, A\&A, 567, A41

Aleksić, J., Ansoldi, S., Antonelli, L. A., et al. 2014b, A\&A, 572, A121

Aleksić, J., Ansoldi, S., Antonelli, L. A., et al. 2015a, J. High Energy Astrophys., 5,30

Aleksić, J., Ansoldi, S., Antonelli, L. A., et al. 2015b, A\&A, 573, A50

Aleksić, J., Ansoldi, S., Antonelli, L. A., et al. 2015c, MNRAS, 446, 217

Aleksić, J., Ansoldi, S., Antonelli, L. A., et al. 2015d, MNRAS, 451, 739

Aleksić, J., Ansoldi, S., Antonelli, L. A., et al. 2016, Astropart. Phys., 72, 76

Aliu, E., Anderhub, H., Antonelli, L. A., et al. 2009, Astropart. Phys., 30, 293

Atwood, W. B., Abdo, A. A., Ackermann, M., et al. 2009, ApJ, 697, 1071

Augusto, P., Wilkinson, P. N., \& Browne, I. W. A. 1998, MNRAS, 299, 1159

Baars, J. W. M., Genzel, R., Pauliny-Toth, I. I. K., \& Witzel, A. 1977, A\&A, 61, 99

Barres de Almeida, U., Ward, M. J., Dominici, T. P., et al. 2010, MNRAS, 408 , 1778

Barthelmy, S. D., Barbier, L. M., Cummings, J. R., et al. 2005, Space Sci. Rev., 120,143

Bessell, M. S. 1979, PASP, 91, 589

Bessell, M. S., Castelli, F., \& Plez, B. 1998, A\&A, 333, 231

Blinov, D., Pavlidou, V., Papadakis, I., et al. 2015, MNRAS, 453, 1669

Burrows, D. N., Hill, J. E., Nousek, J. A., et al. 2005, Space Sci. Rev., 120, 165

Cash, W. 1979, ApJ, 228, 939

Costamante, L., \& Ghisellini, G. 2002, A\&A, 384, 56

Deller, A. T., Brisken, W. F., Phillips, C. J., et al. 2011, PASP, 123, 275

Dermer, C. D., Yan, D., Zhang, L., Finke, J. D., \& Lott, B. 2015, ApJ, 809, 174

Domínguez, A., Primack, J. R., Rosario, D. J., et al. 2011, MNRAS, 410, 2556

Donato, D., Ghisellini, G., Tagliaferri, G., \& Fossati, G. 2001, A\&A, 375, 739

Dondi, L., \& Ghisellini, G. 1995, MNRAS, 273, 583

Fegan, S. J., Badran, H. M., Bond, I. H., et al. 2005, ApJ, 624, 638

Finke, J. D., Dermer, C. D., \& Böttcher, M. 2008, ApJ, 686, 181

Fiorucci, M., Tosti, G., \& Rizzi, N. 1998, PASP, 110, 105

Fitzpatrick, E. L. 1999, PASP, 111, 63

Fomin, V. P., Stepanian, A. A., Lamb, R. C., et al. 1994, Astropart. Phys., 2, 137

Gehrels, N., Chincarini, G., Giommi, P., et al. 2004, ApJ, 611, 1005

Georganopoulos, M., \& Kazanas, D. 2003, ApJ, 594, L27

Ghisellini, G. 2000, in Special Relativity at Action in the Universe, in Recent

Developments in General Relativity, eds. B. Casciaro, D. Fortunato, M.

Francaviglia, \& A. Masiello (Milan: Springer), 5

Ghisellini, G., Tavecchio, F., \& Chiaberge, M. 2005, A\&A, 432, 401

Hillas, A. M. 1985, in Proc. 19th ICRC, La Jolla, ed. F. C. Jones, Int. Cosmic Ray Conference, 3, 445

Humphrey, P. J., Liu, W., \& Buote, D. A. 2009, ApJ, 693, 822

Kalberla, P. M. W., Burton, W. B., Hartmann, D., et al. 2005, A\&A, 440, 775

Katarzyński, K., Sol, H., \& Kus, A. 2001, A\&A, 367, 809

Kneiske, T. M., Mannheim, K., \& Hartmann, D. H. 2002, A\&A, 386, 1

Li, T., \& Ma, Y. 1983, ApJ, 272, 317

Lister, M. L., Cohen, M. H., Homan, D. C., et al. 2009, AJ, 138, 1874

Lister, M. L., Aller, M. F., Aller, H. D., et al. 2013, AJ, 146, 120
Lombardi, S., Berger, K., Colin, P., et al. 2011, in Proc. 32nd ICRC, Beijing, Int. Cosmic Ray Conference, 3, 262

Mannheim, K. 1993, A\&A, 269, 67

Maraschi, L., \& Tavecchio, F. 2003, ApJ, 593, 667

Mattox, J. R., Bertsch, D. L., Chiang, J., et al. 1996, ApJ, 461, 396

Mazin, D., Tescaro, D., Garczarczyk, M., et al. 2013, in Int. Cosmic Ray Conference, Proc. 33rd ICRC, Rio de Janeiro

Mundell, C. G., Kopac, D., Arnold, D. M., et al. 2013, Nature, 504, 119

Nakagawa, A., Edwards, P. G., Murata, Y., Wajima, K., \& Modaka, T. 2005, PASJ, 57, 295

Nilsson, K., Pasanen, M., Takalo, L. O., et al. 2007, A\&A, 475, 199

Nolan, P. L., Abdo, A. A., Ackermann, M., et al. 2012, ApJS, 199, 31

Pacholczyk, A. G. 1970, Radio astrophysics. Nonthermal processes in galactic and extragalactic sources (San Francisco: W. H. Freeman \& Co Ltd)

Padovani, P., \& Giommi, P. 1995, ApJ, 444, 567

Pavlidou, V., Angelakis, E., Myserlis, I., et al. 2014, MNRAS, 442, 1693

Piner, B. G., \& Edwards, P. G. 2013, EPJ Web Conf., 61, 04021

Piner, B. G., Pant, N., \& Edwards, P. G. 2010, ApJ, 723, 1150

Poole, T. S., Breeveld, A. A., Page, M. J., et al. 2008, MNRAS, 383, 627

Poutanen, J., Zdziarski, A. A., \& Ibragimov, A. 2008, MNRAS, 389, 1427

Richards, J. L., Max-Moerbeck, W., Pavlidou, V., et al. 2011, ApJS, 194, 29

Richards, J. L., Hovatta, T., Max-Moerbeck, W., et al. 2014, MNRAS, 438, 3058

Rolke, W. A., López, A. M., \& Conrad, J. 2005, NIMPA, 551, 493

Roming, P. W. A., Kennedy, T. E., Mason, K. O., et al. 2005, Space Sci. Rev., 120,95

Schlafly, E. F., \& Finkbeiner, D. P. 2011, ApJ, 737, 103

Sikora, M., Begelman, M. C., \& Rees, M. J. 1994, ApJ, 421, 153

Sitarek, J., Gaug, M., Mazin, D., Paoletti, R., \& Tescaro, D. 2013, NIPMA, 723, 109

Spergel, D. N., Verde, L., Peiris, H. V., et al. 2003, ApJS, 148, 175

Spurio, M. 2014, Particles and Astrophysics: A Multi-Messenger Approach (Springer Verlag)

Steele, I. A., Bates, S. D., Guidorzi, C., et al. 2010, in SPIE Conf. Ser., 7735

Stickel, M., Fried, J. W., Kühr, H., Padovani, P., \& Urry, C. M. 1991, ApJ, 374, 431

Tavecchio, F., \& Ghisellini, G. 2016, MNRAS, 456, 2374

Tavecchio, F., Maraschi, L., \& Ghisellini, G. 1998, ApJ, 509, 608

Tavecchio, F., Ghisellini, G., Ghirlanda, G., Foschini, L., \& Maraschi, L. 2010, MNRAS, 401, 1570

Teraesranta, H., Tornikoski, M., Mujunen, A., et al. 1998, A\&AS, 132, 305

Tiet, V. C., Piner, B. G., \& Edwards, P. G. 2012, Fermi, \& Jansky Proceedings [arXiv: 1205.2399]

Urry, C. M., \& Padovani, P. 1995, PASP, 107, 803

Vaughan, S., Edelson, R., Warwick, R. S., \& Uttley, P. 2003, MNRAS, 345, 1271

Wisniewski, W. Z., Sitko, M. L., \& Sitko, A. K. 1986, MNRAS, 219, 299

Zanin, R., Carmona, E., Sitarek, J., et al. 2013, in Int. Cosmic Ray Conference, Proc. 33rd ICRC, Rio de Janeiro

1 IFAE, Campus UAB, 08193 Bellaterra, Spain

2 Università di Udine, and INFN Trieste, 33100 Udine, Italy

INAF National Institute for Astrophysics, 00136 Rome, Italy

4 Università di Siena, and INFN Pisa, 53100 Siena, Italy

5 Croatian MAGIC Consortium, Rudjer Boskovic Institute, University of Rijeka and University of Split, 10000 Zagreb, Croatia

6 Max-Planck-Institut für Physik, 80805 München, Germany

7 Universidad Complutense, 28040 Madrid, Spain

8 Inst. de Astrofísica de Canarias, 38200 La Laguna, Tenerife, Spain

9 University of Łódź, 90236 Lodz, Poland

10 Deutsches Elektronen-Synchrotron (DESY), 15738 Zeuthen, Germany

11 ETH Zurich, 8093 Zurich, Switzerland

12 Universität Würzburg, 97074 Würzburg, Germany

13 Centro de Investigaciones Energéticas, Medioambientales y Tecnológicas, 28040 Madrid, Spain

14 Institute of Space Sciences, 08193 Barcelona, Spain

15 Università di Padova and INFN, 35131 Padova, Italy

16 Technische Universität Dortmund, 44221 Dortmund, Germany

17 Unitat de Física de les Radiacions, Departament de Física, and CERES-IEEC, Universitat Autònoma de Barcelona, 08193 Bellaterra, Spain

18 Universitat de Barcelona, ICC, IEEC-UB, 08028 Barcelona, Spain

19 Japanese MAGIC Consortium, KEK, Department of Physics and Hakubi Center, Kyoto University, Tokai University, The University of Tokushima, ICRR, The University of Tokyo, Tokyo, Japan 
${ }^{20}$ Finnish MAGIC Consortium, Tuorla Observatory, University of Turku and Department of Physics, University of Oulu, Oulu, Finland

21 Inst. for Nucl. Research and Nucl. Energy, 1784 Sofia, Bulgaria

22 Università di Pisa, and INFN Pisa, 56126 Pisa, Italy

${ }^{23}$ ICREA and Institute of Space Sciences, 08193 Barcelona, Spain

${ }^{24}$ Università dell'Insubria and INFN Milano Bicocca, Como, 22100 Como, Italy

25 NASA Goddard Space Flight Center, Greenbelt, MD 20771, USA and Department of Physics and Department of Astronomy, University of Maryland, College Park, MD 20742, USA

26 École polytechnique fédérale de Lausanne (EPFL), 1290 Lausanne, Switzerland

27 Institut für Astro- und Teilchenphysik, Leopold-FranzensUniversität Innsbruck, 6020 Innsbruck, Austria

${ }^{28}$ Finnish Centre for Astronomy with ESO (FINCA), Turku, Finland

29 INAF-Trieste, Italy

${ }^{30}$ ISDC - Science Data Center for Astrophysics, 1290 Versoix Geneva, Switzerland

31 Centro Brasileiro de Pesquisas Físicas (CBPF/MCTI),

R. Dr. Xavier Sigaud, 150 - Urca, Rio de Janeiro - RJ, 22290-180, Brazil
2 INAF-IRA Bologna, via Gobetti 101, 40129 Bologna, Italy

33 Aalto University Metsähovi Radio Observatory Kylmälä, Finland

34 Aalto University Dept of Radio Science and Engineering, Espoo, Finland

35 Cahill Center of Astronomy and Astrophysics, California Institute of Technology, 1200 E California Blvd, Pasadena, CA 91125, USA

36 Astro Space Center of Lebedev Physical Institute, Profsoyuznaya 84/32, 117997 Moscow, Russia

37 Max-Planck-Institut für Radioastronomie, Auf dem Hügel 69, 53121 Bonn, Germany

38 Department of Physics, Purdue University, 525 Northwestern Avenue, West Lafayette, IN 47907, USA

39 National Radio Astronomy Observatory, PO Box 0, Socorro, NM 87801, USA

40 Astrophysics Research Institute, Liverpool John Moore University, UK

41 Pulkovo Observatory, Pulkovskoe Chaussee 65/1, 196140 St. Petersburg, Russia

42 Crimean Astrophysical Observatory, 98409 Nauchny, Crimea, Ukraine

43 Laboratoire d'Annecy-le-Vieux de Physique des Particules, Université Savoie Mont-Blanc, CNRS/IN2P3, 74941 Annecy-leVieux, France 